Research Article

\title{
Nonlinear Vibration of the Blade with Variable Thickness
}

\author{
Xiaobo Jie, Wei Zhang $(\mathbb{D}$, and Jiajia Mao \\ College of Mechanical Engineering, Beijing University of Technology, \\ Beijing Key Laboratory of Nonlinear Vibrations and Strength of Mechanical Structures, Beijing 100124, China
}

Correspondence should be addressed to Wei Zhang; sandyzhang0@yahoo.com

Received 13 January 2020; Revised 2 March 2020; Accepted 16 March 2020; Published 9 April 2020

Academic Editor: Denis Benasciutti

Copyright (c) 2020 Xiaobo Jie et al. This is an open access article distributed under the Creative Commons Attribution License, which permits unrestricted use, distribution, and reproduction in any medium, provided the original work is properly cited.

In this paper, the nonlinear dynamic responses of the blade with variable thickness are investigated by simulating it as a rotating pretwisted cantilever conical shell with variable thickness. The governing equations of motion are derived based on the von Kármán nonlinear relationship, Hamilton's principle, and the first-order shear deformation theory. Galerkin's method is employed to transform the partial differential governing equations of motion to a set of nonlinear ordinary differential equations. Then, some important numerical results are presented in terms of significant input parameters.

\section{Introduction}

Rotating blades can be found in different engineering problems as the key component of aircraft engines. Certain important design considerations require a thorough understanding of the structural dynamic characteristics of rotating blades. Extensive uses of pretwisted cantilever conical shells can be found in different engineering disciplines. The dynamic behaviors of the shell are significant for the analysis of the whole structure of blades.

The vibrations of different kinds of shells have been studied. Qinkai and Fulei [1] analyzed the instability of a rotating truncated conical shell subjected to periodic axial loads. Malekzadeh and Heydarpour [2] investigated the free vibration behaviors of rotating FG truncated conical shells with different edge conditions. Goldberg et al. [3] and Yang [4] analyzed the vibration of the conical shell by numerical integration. Srinivasan and Krishnan [5] presented an analysis of the dynamic behaviors for the conical panel using an integral equation technique. Liew et al. [6] and McGee and Chu [7] have investigated the vibration characteristics of pretwisted conical shells by the Ritz method while Lee et al. [8] have analyzed the vibration characteristics of twisted cantilevered conical composite shells by using the finite element method. Naidu and Sinha [9] studied the nonlinear free vibration of laminated composite shells in hygrothermal environment incorporating the Green-Lagrange-type nonlinear strains. Kumari and Sinha [10] demonstrated the hygrothermal effects on the behavior of composite $\mathrm{T}$-joints using a modified thick shell element. Ghosh [11] analyzed the hygrothermal effects on the initiation and propagation of damage in composite shells due to low-velocity impacts. Bandyopardhyay et al. [12] investigated the hygrothermal effects on the free vibration characteristics of delaminated carbon-epoxy composite pretwisted rotating conical shells. Hwu et al. [13] presented a modified FSDT model for obtaining closed-form solutions of natural frequencies for certain particular problems of sandwich plates and shells. Garg et al. [14] presented the closed form solutions based on the HSDT to study the free vibration characteristics of doubly curved sandwich shells. Rahmani et al. [15] proposed a sandwich plate theory that employs the FSDT for composite face-sheets and the HSDT for flexible cores to study the free vibration characteristics of sandwich cylindrical shells. Kumar et al. [16] employed an efficient FE model based on the higher-order zig-zag theory to solve the free vibration problem of multilayered composites and sandwich shallow shells. The study of free vibration characteristics of isotropic and multilayered composite shallow conical shells with pretwists have been carried out by Lim et al. [17] and Lee et al. [8] using the FSDT. Sofyev [18] investigated the free vibration characteristics of laminated orthotropic conical shells using the modified FSDT. The vibration and buckling of functionally graded material (FGM) conical shells using 
various theories has been reported by Sofyev [19]. Wilkins et al. [20] investigated the symmetrical and unsymmetrical vibration modes of sandwich conical shells with various boundary conditions. Bardell et al. [21] developed an FE model to study the vibration characteristics of sandwich conical panels and carried out an experiment to verify the model. Nasihatgozar and Khalili [22] analyzed the vibration and buckling of sandwich truncated conical shells using the differential quadrature method. Sofyev and Osmancelebioglu [23] studied the free vibration behavior of sandwich truncated conical shells containing FGM coatings using the FSDT. Deb Singha et al. [24] studied the influence of elevated temperature and moisture absorption on the free vibration behavior of rotating pretwisted sandwich conical shells consisting of two composite face sheets and a synthetic foam core. The stress, deformation, and snap-through conditions of thin, axisymmetric, shallow bimetallic shells were investigated by Jakomin and Kosel [25]. da Silva et al. [26] studied the influence of Young's modulus, shell thickness, and geometrical imperfection uncertainties on the parametric instability loads of simply supported axially excited cylindrical shells. Li et al. [27] had a research on the free vibration of the stiffened cylindrical shell with general boundary conditions.

The vibration of the cantilever blade with variable thickness has been less studied because of the mathematical complexity in describing the geometry and nonlinear dynamic behaviors. Thermoelastic behavior of a functionally graded blade with variable thickness subjected to the mechanical and thermal loadings has been investigated by Mirzaei et al. [28, 29].

In this paper, the nonlinear dynamic responses of the blade with variable thickness are investigated by simulating it as a rotating pretwisted cantilever conical shell with variable thickness. The governing equations of motion are derived based on the von Kármán nonlinear relationship, Hamilton's principle, and the first-order shear deformation theory. Galerkin's method is employed to transform the partial differential governing equations of motion to a set of nonlinear ordinary differential equations. Then, the effects of the varying rotating speed, external force, and external moment on the dynamic behavior of the blade are studied.

\section{Theoretical Formulations}

2.1. Description of Blade. As shown in Figure 1, the blade is regarded as a pretwisted thin-walled rotating cantilever conical shell with varying rotating speed and the thickness. The coordinate system $(x, \theta$, and $z)$ is located in the middle surface of the pretwisted thin-walled cantilever conical shell. The coordinate components $(u, v$, and $w)$ represent the displacements of an arbitrary point in the $x$, $\theta$, and $z$ directions, respectively. The geometrical sizes of the pretwisted thin-walled cantilever conical shell are represented by the bottom inner radius $r_{\text {root }}$, subtended angle $\alpha$, presetting angle $\beta$, pretwisted angle $\Phi$, semivertex angle $\psi$, length $L$, and varying thickness $h=h_{0}(x) g(\theta)$, respectively. Therefore, we have the radius at any point along the length:

$$
R=r_{\text {root }}-x \tan \psi
$$

Other geometric parameters of the pretwisted thinwalled cantilever conical shell are given as

$$
\begin{aligned}
h_{0}(x) & =\max \{h(x)\}, \\
g(\theta) & =\frac{f(\theta)}{a}, \\
a & =\max \{f(\theta)\}, \\
f(\theta) & =\left(\sin \frac{\alpha}{2}-\sin \theta\right)^{(3 / 2)}\left(\sin \frac{\alpha}{2}-\sin \theta\right)^{(1 / 2)}, \\
\theta & \in\left[-\frac{\alpha}{2}, \frac{\alpha}{2}\right] .
\end{aligned}
$$

The pretwisted blade, which is installed on a rigid hub with the radius $r_{0}$, rotates at the speed $\Omega(t)=\Omega_{c}+\Omega_{v} \cos \omega_{r} t$ about its polar axis [30, 31]. For the reason that the distribution of the load on the blade varies during one rotation, the load on the rotating blade is periodic and can be described as the force $F=F_{0}+$ $F_{1} \cos \omega_{r} t$ and moment $M=M_{0}+M_{1} \cos \omega_{r} t$ on the upper surface of the pretwisted thin-walled cantilever conical shell. Both of the force $F$ and the moment $M$ include a mean and a simple harmonic component [32].

\subsection{Displacement Field and Geometric Equation.} According to the first-order shear deformation theory, the displacement field $(u, v$, and $w)$ of the pretwisted thin-walled cantilever conical shell is written as

$$
\begin{aligned}
& u(x, \theta, z, t)=u_{0}(x, \theta, t)+z \varphi_{x}(x, \theta, t), \\
& v(x, \theta, z, t)=v_{0}(x, \theta, t)+z \varphi_{\theta}(x, \theta, t), \\
& w(x, \theta, z, t)=w_{0}(x, \theta, t),
\end{aligned}
$$

where $\left(u_{0}, v_{0}, w_{0}\right)$ are, respectively, the midplane displacements in the direction of $x, \theta$, and $z$ for the pretwisted thinwalled cantilever conical shell and $\varphi_{x}$ and $\varphi_{\theta}$ represent the midplane rotations of the transverse normal about the $\theta$ and $x$ axes, respectively.

The strain components $\left(\varepsilon_{x}, \varepsilon_{\theta}, \gamma_{x \theta}\right)$ at an arbitrary point of the pretwisted thin-walled cantilever conical shell are related to the middle surface strains $\left(\varepsilon_{x}^{(0)}, \varepsilon_{\theta}^{(0)}, \gamma_{x \theta}^{(0)}\right)$ and to the changes in the curvature and the torsion of the middle surface $\left(\varepsilon_{x}^{(1)}, \varepsilon_{\theta}^{(1)}, \gamma_{x \theta}^{(1)}\right)$. Using von Karman geometric straindisplacement relations, the expressions [33] can be obtained as

$$
\left[\begin{array}{c}
\varepsilon_{x} \\
\varepsilon_{\theta} \\
\gamma_{x \theta}
\end{array}\right]=\left[\begin{array}{c}
\varepsilon_{x}^{(0)} \\
\varepsilon_{\theta}^{(0)} \\
\gamma_{x \theta}^{(0)}
\end{array}\right]+z\left[\begin{array}{c}
\varepsilon_{x}^{(1)} \\
\varepsilon_{\theta}^{(1)} \\
\gamma_{x \theta}^{(1)}
\end{array}\right],
$$

where 


$$
\begin{aligned}
& {\left[\begin{array}{c}
\varepsilon_{x}^{(0)} \\
\varepsilon_{\theta}^{(0)} \\
\gamma_{x \theta}^{(0)}
\end{array}\right]=\left[\begin{array}{c}
\frac{\partial u_{0}}{\partial x}+\frac{1}{2}\left(\frac{\partial w_{0}}{\partial x}\right)^{2} \\
\frac{1}{R \cos \psi} \frac{\partial v_{0}}{\partial \theta}+\frac{1}{R} w_{0}+\frac{1}{R} u_{0} \tan \psi+\frac{1}{2} \frac{1}{R^{2} \cos ^{2} \psi}\left(\frac{\partial w_{0}}{\partial \theta}\right)^{2} \\
\frac{1}{R \cos \psi} \frac{\partial u_{0}}{\partial \theta}-\frac{1}{R} v_{0} \tan \psi+\frac{\partial v_{0}}{\partial x}+\frac{1}{R \cos \psi} \frac{\partial w_{0}}{\partial x} \frac{\partial w_{0}}{\partial \theta}
\end{array}\right],} \\
& {\left[\begin{array}{c}
\varepsilon_{x}^{(1)} \\
\varepsilon_{\theta}^{(1)} \\
\gamma_{x \theta}^{(1)}
\end{array}\right]=\left[\begin{array}{c}
\frac{\partial \varphi_{x}}{\partial x} \\
\frac{1}{R \cos \psi} \frac{\partial \varphi_{\theta}}{\partial \theta}+\frac{1}{R} \varphi_{x} \tan \psi \\
\frac{1}{R \cos \psi} \frac{\partial \varphi_{x}}{\partial \theta}-\frac{1}{R} \varphi_{\theta} \tan \psi+\frac{\partial \varphi_{\theta}}{\partial x}
\end{array}\right],} \\
& {\left[\begin{array}{c}
\gamma_{\theta z} \\
\gamma_{x z}
\end{array}\right]=\left[\begin{array}{c}
\varphi_{\theta}+\frac{1}{R \cos \psi} \frac{\partial w_{0}}{\partial \theta}-\frac{1}{R} v_{0} \\
\frac{\partial w_{0}}{\partial x}+\varphi_{x}
\end{array}\right]}
\end{aligned}
$$

2.3. Constitutive Relation and Internal Force Relation. The stress-strain relations of the conical shell are expressed as

$$
\left[\begin{array}{c}
\sigma_{x} \\
\sigma_{\theta} \\
\sigma_{x \theta} \\
\sigma_{\theta z} \\
\sigma_{x z}
\end{array}\right]=\left[\begin{array}{lllll}
Q_{11} & Q_{12} & & & \\
Q_{12} & Q_{22} & & & \\
& & Q_{66} & & \\
& & & Q_{44} & \\
& & & & Q_{55}
\end{array}\right]\left[\begin{array}{c}
\varepsilon_{x} \\
\varepsilon_{\theta} \\
\gamma_{x \theta} \\
\gamma_{\theta z} \\
\gamma_{x z}
\end{array}\right],
$$

where $Q_{m n}(m, n=1,2,4,5,6)$ are the stiffness coefficient,

$$
\begin{aligned}
& Q_{11}=Q_{22}=\frac{E}{1-v^{2}}, \\
& Q_{12}=\frac{E v}{1-v^{2}}, \\
& Q_{44}=Q_{55}=Q_{66}=\frac{E}{2(1+\nu)},
\end{aligned}
$$

where $E$ denotes Young's modulus and $v$ is Poisson's ratio.

2.4. Governing Equations of Motion. The Hamilton principle

$$
\int_{0}^{t}\left(\delta u_{1}+\delta u_{2}+\delta w-\delta K\right) \mathrm{d} t=0
$$

is employed to derive the governing equations of motion for the pretwisted thin-walled cantilever conical shell with variable thickness, where $K, u_{1}, u_{2}$, and $W$ denote the kinetic energy, the strain energy, the centrifugal force potential energy, and the virtual work of external forces, respectively, $t$ represents time, and $\delta$ is the variation operator [34].

According to research given in Tong et al. [35] and Konig's theorem, the energy relationship between the pretwisted thinwalled rotating cantilever conical shell with variable thickness and that with constant thickness is described as

$$
\frac{K}{K_{h_{0}}}=\frac{(1 / 2) m r_{c}^{2} \Omega^{2}+o\left((1 / 2) m r_{c}^{2} \Omega^{2}\right)}{(1 / 2) m_{h_{0}} r_{h_{0} c}^{2} \Omega^{2}+o\left((1 / 2) m_{h_{0}} r_{h_{0} c}^{2} \Omega^{2}\right)}
$$

$$
\begin{aligned}
& \approx \frac{m}{m_{h_{0}}}=\frac{1}{\alpha} \int_{-(\alpha / 2)}^{(\alpha / 2)} f(\theta) \mathrm{d} \theta=K_{K}, \\
& \frac{u_{2}}{u_{2 h_{0}}}=\frac{-(1 / 2) m r_{c}^{2} \Omega^{2}}{-(1 / 2) m_{h_{0}} r_{h_{0} c}^{2} \Omega^{2}} \\
& \approx \frac{m}{m_{h_{0}}}=\frac{1}{\alpha} \int_{-(\alpha / 2)}^{(\alpha / 2)} f(\theta) \mathrm{d} \theta=K_{u_{2}},
\end{aligned}
$$

$$
\begin{gathered}
\frac{U_{1}}{U_{1 h_{0}}}=K_{U_{1}}, \\
\frac{W}{W_{h_{0}}}=K_{W},
\end{gathered}
$$

where $K_{h_{0}}, u_{1 h_{0}}, u_{2 h_{0}}$, and $w_{h_{0}}$ are, respectively, the kinetic energy, the strain energy, the centrifugal force potential energy, and the virtual work of the conical shell with the constant thickness $h_{0}$ and $K_{K}, K_{u_{1}}, K_{u_{2}}$, and $K_{w}$ are the constant coefficients. 

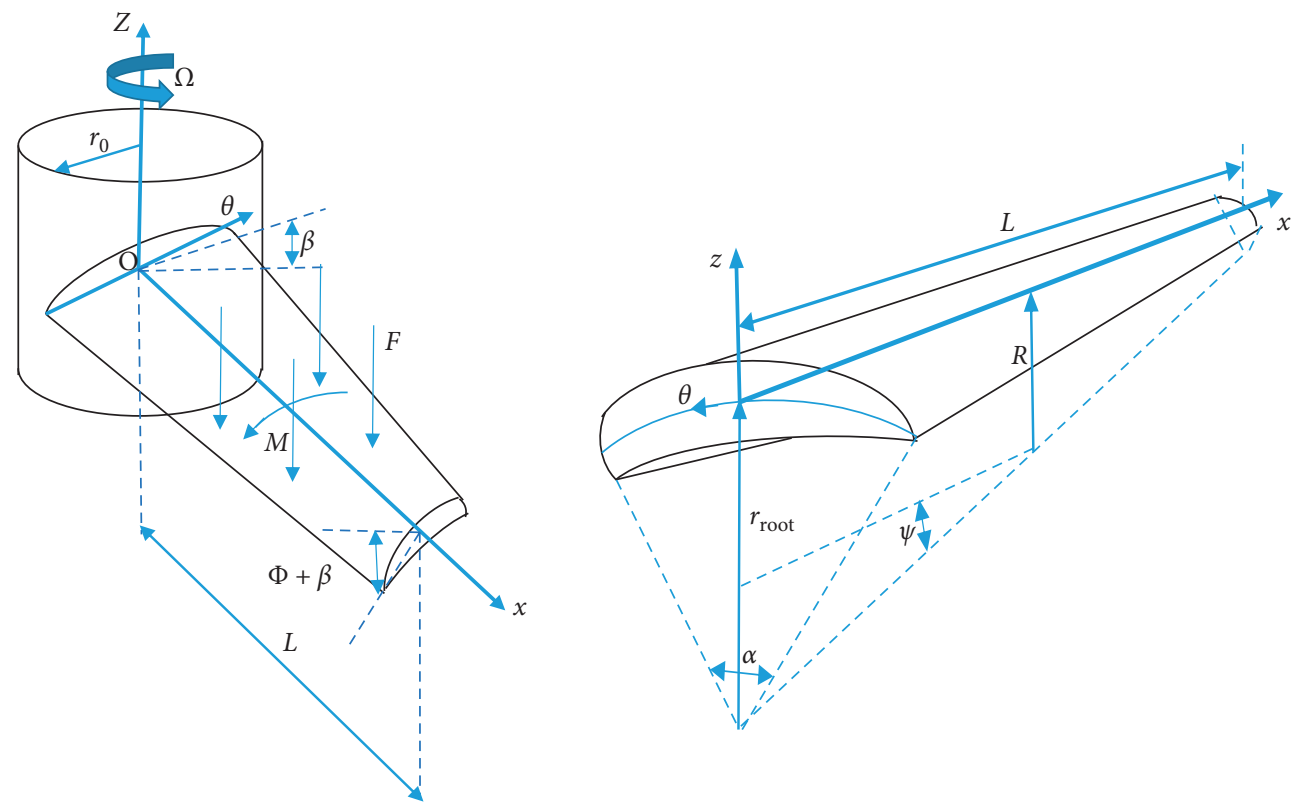

FIGURE 1: The model of presetting and pretwisted cantilever conical shell with varying thickness.

The inertias of the pretwisted thin-walled cantilever conical shell $I_{i}$ are calculated by

$$
I_{i}=\int_{-\left(h_{0} / 2\right)}^{\left(h_{0} / 2\right)} \rho z^{i} \mathrm{~d} z, \quad i=0,1,2,3,4 .
$$

The force and moment resultants are given as

$$
\left[\begin{array}{l}
N_{x x} \\
N_{\theta \theta} \\
N_{x \theta}
\end{array}\right]=\left[\begin{array}{lll}
A_{11} & A_{12} & \\
A_{12} & A_{22} & \\
& & A_{66}
\end{array}\right]\left[\begin{array}{c}
\varepsilon_{x}^{(0)} \\
\varepsilon_{\theta}^{(0)} \\
\gamma_{x \theta}^{(0)}
\end{array}\right]+\left[\begin{array}{lll}
B_{11} & B_{12} & \\
B_{12} & B_{22} & \\
& & B_{66}
\end{array}\right]\left[\begin{array}{c}
\varepsilon_{x}^{(1)} \\
\varepsilon_{\theta}^{(1)} \\
\gamma_{x \theta}^{(1)}
\end{array}\right],
$$

$$
\begin{aligned}
& {\left[\begin{array}{l}
M_{x x} \\
M_{\theta \theta} \\
M_{x \theta}
\end{array}\right]=\left[\begin{array}{lll}
B_{11} & B_{12} & \\
B_{12} & B_{22} & \\
& & B_{66}
\end{array}\right]\left[\begin{array}{c}
\varepsilon_{x}^{(0)} \\
\varepsilon_{\theta}^{(0)} \\
\gamma_{x \theta}^{(0)}
\end{array}\right]+\left[\begin{array}{lll}
D_{11} & D_{12} & \\
D_{12} & D_{22} & \\
& & D_{66}
\end{array}\right]\left[\begin{array}{c}
\varepsilon_{x}^{(1)} \\
\varepsilon_{\theta}^{(1)} \\
\gamma_{x \theta}^{(1)}
\end{array}\right],} \\
& {\left[\begin{array}{l}
Q_{x} \\
Q_{\theta}
\end{array}\right]=\left[\begin{array}{ll}
A_{44} & \\
& A_{55}
\end{array}\right]\left[\begin{array}{l}
\gamma_{x z} \\
\gamma_{\theta z}
\end{array}\right],}
\end{aligned}
$$

where $A_{\mathrm{ij}}, B_{\mathrm{ij}}$, and $D_{\mathrm{ij}}$ are, respectively, called the extensional, the bending-extensional coupling, and the bending stiffness terms,

$$
\begin{aligned}
\left(A_{\mathrm{ij}}, B_{\mathrm{ij}}, D_{\mathrm{ij}}\right) & =\int_{-\left(h_{0} / 2\right)}^{\left(h_{0} / 2\right)} \mathrm{Q}_{\mathrm{ij}}\left(1, z, z^{2}\right) \mathrm{d} z, \quad i, j=1,2,6, \\
A_{\mathrm{ij}} & =\int_{-\left(h_{0} / 2\right)}^{\left(h_{0} / 2\right)} \mathrm{Q}_{\mathrm{ij}} \mathrm{d} z, \quad i, j=4,5 .
\end{aligned}
$$

With one end clamped and others free, the boundary conditions of the pretwisted thin-walled rotating cantilever conical shell are written as

$$
\begin{aligned}
u_{0} & =v_{0}=w_{0}=\varphi_{x}=\varphi_{\theta}=0, \quad x=0, \\
N_{x x} & =N_{\theta \theta}=N_{x \theta}=M_{x x}=M_{\theta \theta}=M_{x \theta}=Q_{x} \\
& =Q_{\theta}=0, \quad x=L, \\
N_{x x} & =N_{\theta \theta}=N_{x \theta}=M_{x x}=M_{\theta \theta}=M_{x \theta} \\
& =Q_{x}=Q_{\theta}=0, \quad \theta=-\frac{\pi}{2} \text { and } \frac{\pi}{2} .
\end{aligned}
$$

In general, the first two-mode truncation is accurate enough to analyze the dynamics response as the lower frequencies often play the most important role in the nonlinear dynamics systems [30,31]. Hence, we focus on the first two modes. The approximate mode functions are

$$
\begin{aligned}
u_{0}(x, \theta, t)= & u_{1}(t) \sin \left(\frac{\pi}{L} x\right) \cos (3 \theta-3 x) \\
& +u_{2}(t) \sin \left(\frac{3 \pi}{L} x\right) \cos (\theta-x), \\
v_{0}(x, \theta, t)= & v_{1}(t) \sin \left(\frac{\pi}{L} x\right) \sin (3 \theta-3 x) \\
& +v_{2}(t) \sin \left(\frac{3 \pi}{L} x\right) \sin (\theta-x), \\
w_{0}(x, \theta, t)= & w_{1}(t) \sin \left(\frac{\pi}{L} x\right) \cos (3 \theta-3 x) \\
& +w_{2}(t) \sin \left(\frac{3 \pi}{L} x\right) \cos (\theta-x),
\end{aligned}
$$




$$
\begin{gathered}
\varphi_{x}(x, \theta, t)=\varphi_{x 1}(t) \sin \left(\frac{\pi}{L} x\right) \cos (3 \theta-3 x) \\
+\varphi_{x 2}(t) \sin \left(\frac{3 \pi}{L} x\right) \cos (\theta-x), \\
\varphi_{\theta}(x, \theta, t)=\varphi_{\theta 1}(t) \sin \left(\frac{\pi}{L} x\right) \sin (3 \theta-3 x) \\
+\varphi_{\theta 2}(t) \sin \left(\frac{3 \pi}{L} x\right) \sin (\theta-x), \\
F_{1}=F_{11} \sin \left(\frac{\pi}{L} x\right) \cos (3 \theta-3 x)+F_{22} \sin \left(\frac{3 \pi}{L} x\right) \cos (\theta-x), \\
M_{1}=M_{11} \sin \left(\frac{\pi}{L} x\right) \sin (3 \theta-3 x)+M_{22} \sin \left(\frac{3 \pi}{L} x\right) \sin (\theta-x) .
\end{gathered}
$$

Using the equations above, the nonlinear equations of motion in terms of generalized displacements can be obtained. All the inertia terms of $u_{0}, v_{0}$, and $\varphi_{x}$ in nonlinear equations of motion can be ignored since their influences are small compared to the inertia terms of $w_{0}$ and $\varphi_{\theta}$ [34]. Then, we can derive the displacements $u_{0}, v_{0}$, and $\varphi_{x}$ with respect to the displacement $w_{0}$ and $\varphi_{\theta}$. In the following analysis, we express $u_{0}, v_{0}$, and $\varphi_{x}$ in terms of $w_{1}, w_{2}, \varphi_{\theta 1}$, and $\varphi_{\theta 2}$. Finally the nondimensional nonlinear system is obtained:

$$
\begin{aligned}
\ddot{w}_{1}+ & \mu_{1} \dot{w}_{1}+\omega_{1}^{2} w_{1}+\alpha_{1} w_{1} \cos \omega_{r} t \\
& +\alpha_{2} w_{1}\left(\cos \omega_{r} t\right)^{2}+\alpha_{3} w_{1}^{2}+\alpha_{4} w_{2}^{2}+\alpha_{5} w_{1} w_{2} \\
& +\alpha_{6} w_{1}^{2} w_{2}+\alpha_{7} w_{1}^{3}+\alpha_{8} w_{2}^{3}+\alpha_{9} \varphi_{\theta 1} \\
& +\alpha_{10} \varphi_{\theta 1} \cos \omega_{r} t+\alpha_{11} w_{1} \varphi_{\theta 2}+\alpha_{12} \varphi_{\theta 1}\left(\cos \omega_{r} t\right)^{2} \\
& +\alpha_{13} w_{1} \varphi_{\theta 1}+\alpha_{14} w_{2} \varphi_{\theta 1}+\alpha_{15} w_{2} \varphi_{\theta 2} \\
= & \alpha_{16}+f_{1} \cos \omega_{r} t \\
& \\
\ddot{w}_{2}+ & \mu_{2} \dot{w}_{2}+\omega_{2}^{2} w_{2}+\beta_{1} w_{2} \cos \omega_{r} t \\
& +\beta_{2} w_{2}\left(\cos \omega_{r} t\right)^{2}+\beta_{3} w_{1}^{2}+\beta_{4} w_{2}^{2}+\beta_{5} w_{1} w_{2} \\
& +\beta_{6} w_{1}^{2} w_{2}+\beta_{7} w_{1}^{3}+\beta_{8} w_{2}^{3}+\beta_{9} \varphi_{\theta 2} \\
& +\beta_{10} \varphi_{\theta 2} \cos \omega_{r} t+\beta_{11} w_{1} \varphi_{\theta 2} \\
& +\beta_{12} \varphi_{\theta 2}\left(\cos \omega_{r} t\right)^{2}+\beta_{13} w_{1} \varphi_{\theta 1}+\beta_{14} w_{2} \varphi_{\theta 1} \\
& +\beta_{15} w_{2} \varphi_{\theta 2} \\
= & \beta_{16}+f_{2} \cos \omega_{r} t \\
\varphi_{\theta 1}+ & \mu_{3} \dot{\varphi}_{\theta 1}+\omega_{3}^{2} \varphi_{\theta 1}+\gamma_{1} \varphi_{\theta 1} \cos \omega_{r} t \\
& +\gamma_{2} \varphi_{\theta 1}\left(\cos \omega_{r} t\right)^{2}+\gamma_{3} w_{1}+\gamma_{4} w_{1} \cos \omega_{r} t \\
& +\gamma_{5} w_{1}\left(\cos \omega_{r} t\right)^{2}+\gamma_{6} w_{1}^{2}+\gamma_{7} w_{1} w_{2}+\gamma_{8} w_{2}^{2} \\
= & \gamma_{9}+m_{1} \cos \omega_{r} t, \\
&
\end{aligned}
$$

$$
\begin{aligned}
\varphi_{\theta 2}+ & \mu_{4} \dot{\varphi}_{\theta 2}+\omega_{4}^{2} \varphi_{\theta 2}+\eta_{1} \varphi_{\theta 2} \cos \omega_{r} t \\
& +\eta_{2} \varphi_{\theta 2}\left(\cos \omega_{r} t\right)^{2}+\eta_{3} w_{1}+\eta_{4} w_{1} \cos \omega_{r} t \\
& +\eta_{5} w_{1}\left(\cos \omega_{r} t\right)^{2}+\eta_{6} w_{1}^{2}+\eta_{7} w_{1} w_{2} \\
& +\eta_{8} w_{2}^{2} \\
= & \eta_{9}+m_{2} \cos \omega_{r} t .
\end{aligned}
$$

\section{Numerical Simulation}

The nonlinear dynamic responses of the rotating cantilever conical shell under different rotating velocities and different excitations are studied in this part through some numerical simulations. To analyze the chaotic and periodic motions in the system, the phase portraits, time history diagrams, threedimensional phase portraits, and power spectrum densities (PSD) are obtained.

3.1. Validation. Based on the kinetic energy and the potential energy of the blade deduced in the previous section, the Chebyshev polynomial and Ritz method are used to calculate the frequency of the model, which are compared with the frequency obtained by the finite element method.

The Chebyshev polynomial series $P_{i}(\chi)$ and $P_{j}(\chi) \quad(i=$ $1,2, \ldots ; J=1,2, \ldots)$ are complete and orthogonal sets. The polynomial terms are taken as $i \times J=10 \times 10$, where $I$ and $J$ are the truncation orders of the Chebyshev polynomial series.

The finite element analysis type is a modal analysis with the Block Lanczos method (using Ansys 15.0). The unit type is SOLID 95 with 20 nodes, and we adopt an intellectual finite element mesh division with four-stage precision for the mesh division.

3.1.1. The Comparative Analysis of Frequencies Using the Chebyshev-Ritz Method, the Finite Element Method, and a Modal Experiment. Completely free edge conditions are selected because they are the most exacting proof of any proposed theoretical solution scheme. And this work would provide a valuable reference datum for the subsequent theoretical study

To validate the accuracy of the frequencies worked out by the Chebyshev-Ritz method, we compare frequencies analytically calculated, with the frequencies obtained by the finite element method, the results from Ye et al. [36] and the datum from an experiment by Bardell et al. [21]. C-R represents the frequencies solved by the Chebyshev-Ritz method, FEM represents the frequencies obtained by the finite element method, and EX represents the frequencies by experiment.

Parameters of the blade are selected as 
TABLE 1: Frequency comparison.

\begin{tabular}{|c|c|c|c|c|c|c|}
\hline & \multicolumn{3}{|c|}{$1^{\text {st }}$} & \multicolumn{3}{|c|}{ 2nd } \\
\hline & $\mathrm{C}-\mathrm{R}$ & FEM & EX & $\mathrm{C}-\mathrm{R}$ & FEM & EX \\
\hline Bardell et al. [21] & & 4.31 & 4.5 & & 8.58 & 8.9 \\
\hline Ye et al. [36] & 4.27 & & & 8.20 & & \\
\hline Present & 4.26 & 4.33 & & 8.21 & 8.59 & \\
\hline
\end{tabular}

TABle 2: Frequency comparison.

\begin{tabular}{|c|c|c|c|c|}
\hline & \multicolumn{2}{|c|}{$1^{\text {st }}$} & \multicolumn{2}{|c|}{ 2nd } \\
\hline & C-R & FEM & C-R & FEM \\
\hline $0 \mathrm{rpm}$ & 1187.3 & 1243.5 & 1779.2 & 1935.1 \\
\hline $500 \mathrm{rpm}$ & 1199.7 & 1252.2 & 1790.6 & 1945.4 \\
\hline
\end{tabular}

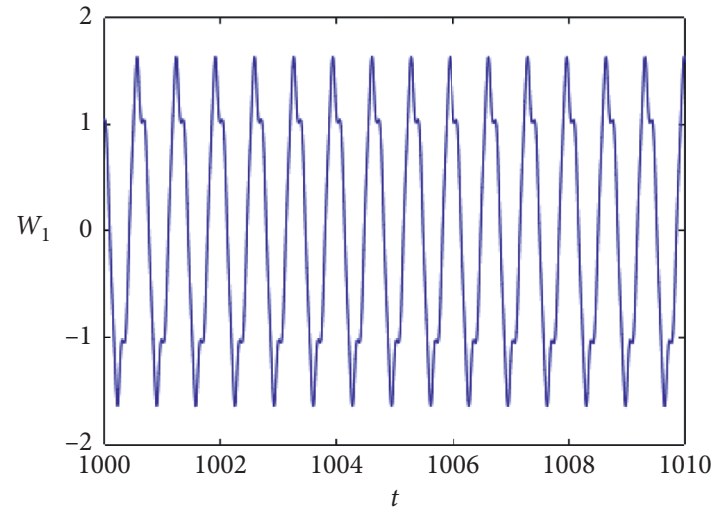

(a)

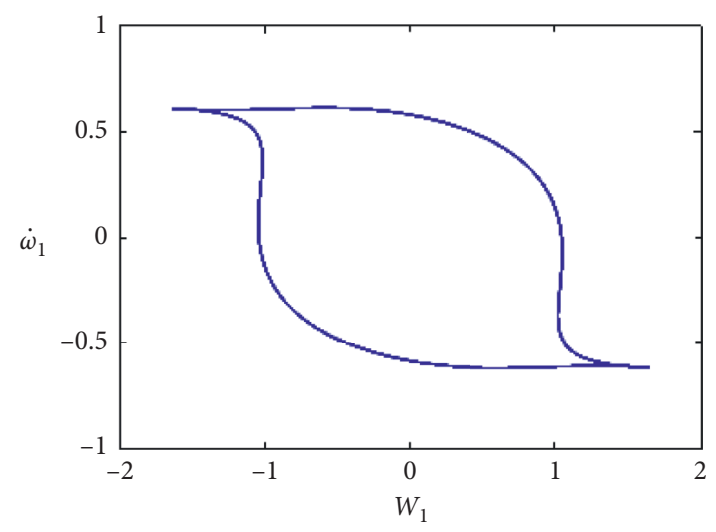

(c)

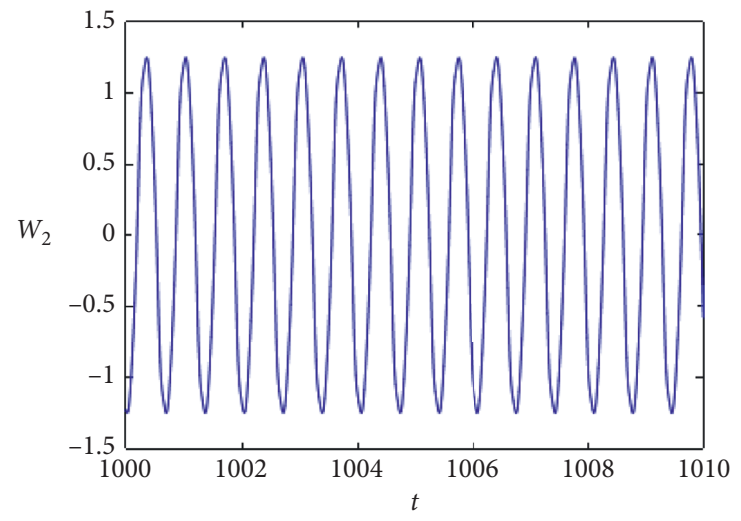

(b)

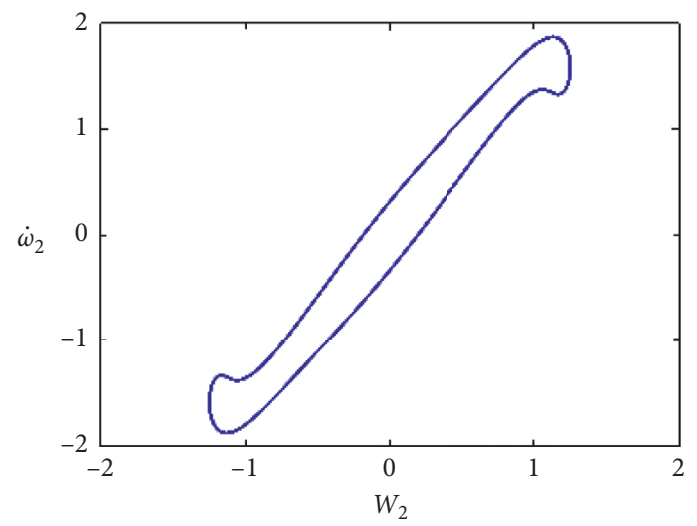

(d)

FIgUre 2: Continued. 


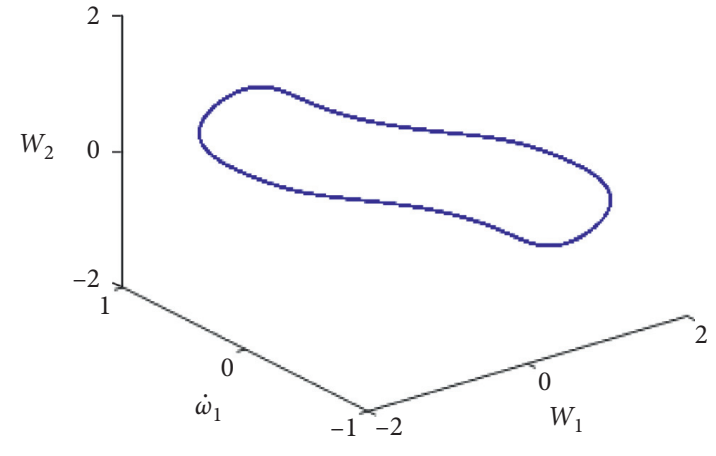

(e)

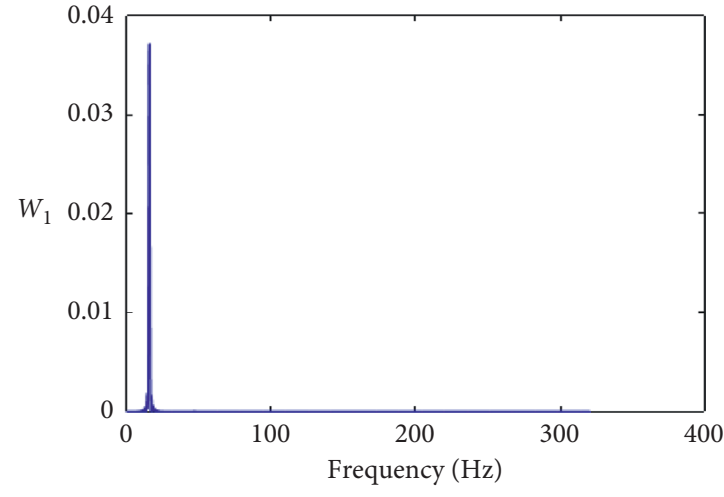

(f)

Figure 2: The motion of the blade with varying rotating speeds when $\Omega_{v}=0.17 \times 10^{2}(\mathrm{r} / \mathrm{min})$. The time history of the first-order mode of (a) $w_{1}$ and (b) $w_{2}$; the phase portrait (c) on plane $\left(w_{1}, \dot{w}_{1}\right)$, (d) on plane $\left(w_{2}, \dot{w}_{2}\right)$, and (e) in the three-dimensional space $\left(w_{1}, \dot{w}_{1}, w_{2}\right)$; and (f) the PSD of $w_{1}$.

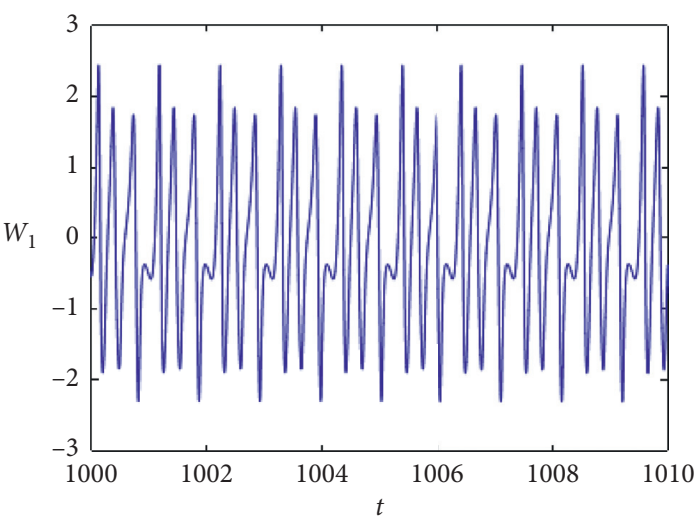

(a)

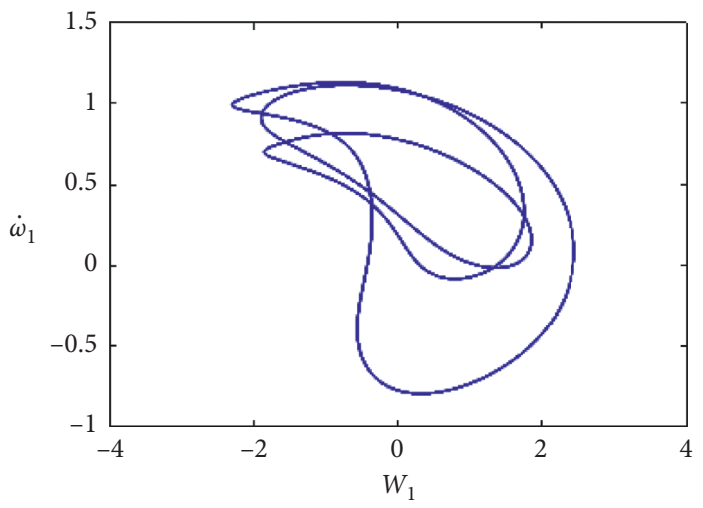

(c)

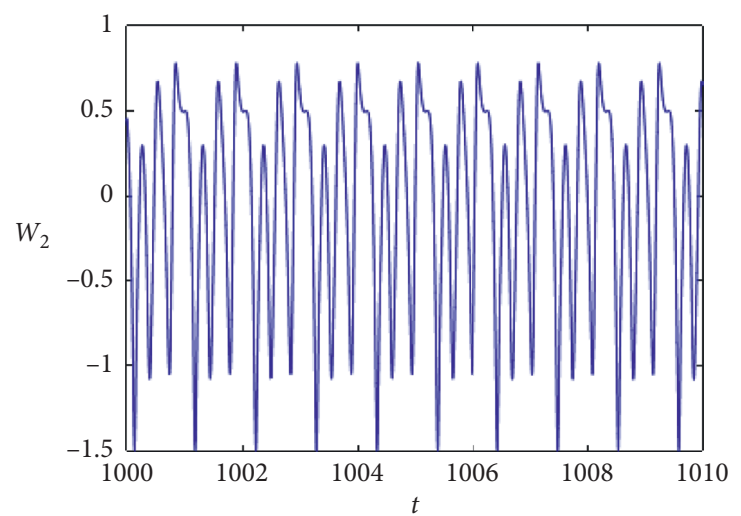

(b)

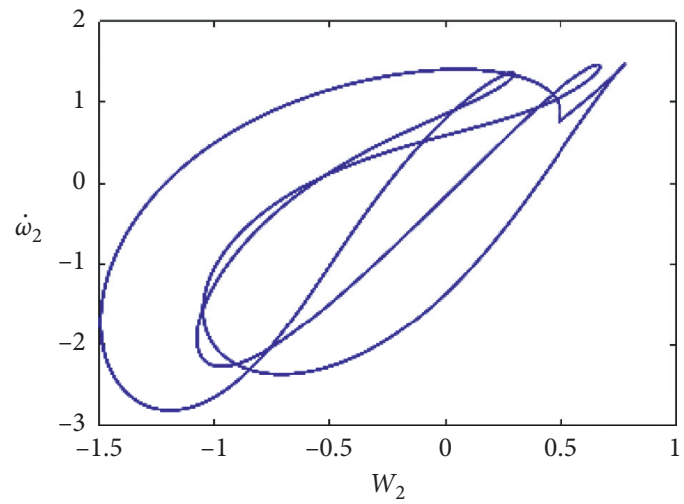

(d)

Figure 3: Continued. 


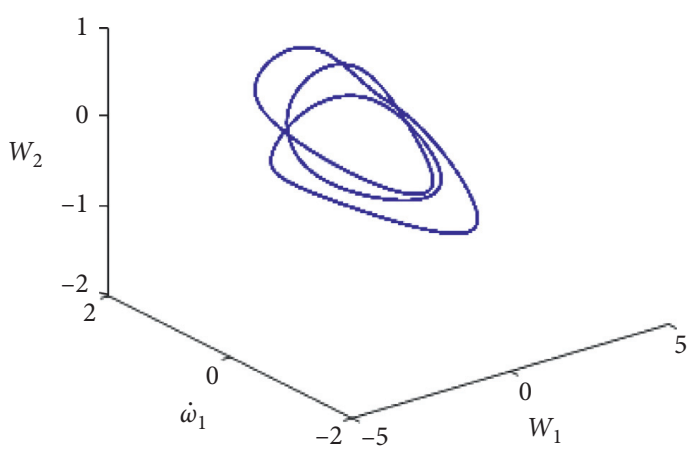

(e)

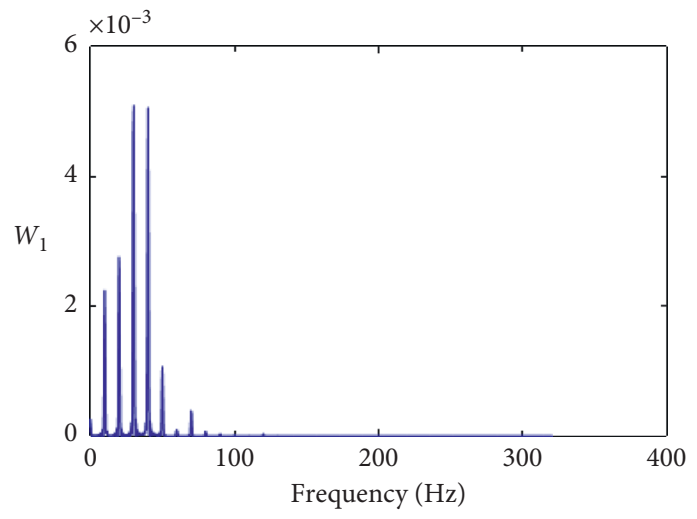

(f)

FIGURE 3: The motion of the blade with varying rotating speeds when $\Omega_{v}=1.09 \times 10^{2}(\mathrm{r} / \mathrm{min})$. The time history of the first-order mode of (a) $w_{1}$ and (b) $w_{2}$; the phase portrait (c) on plane $\left(w_{1}, \dot{w}_{1}\right)$, (d) on plane $\left(w_{2}, \dot{w}_{2}\right)$, and (e) in the three-dimensional space $\left(w_{1}, \dot{w}_{1}, w_{2}\right)$; and (f) the PSD of $w_{1}$.

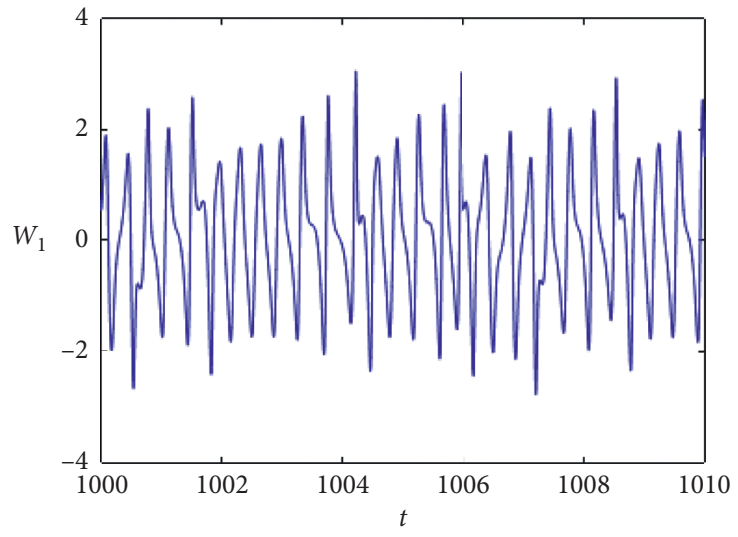

(a)

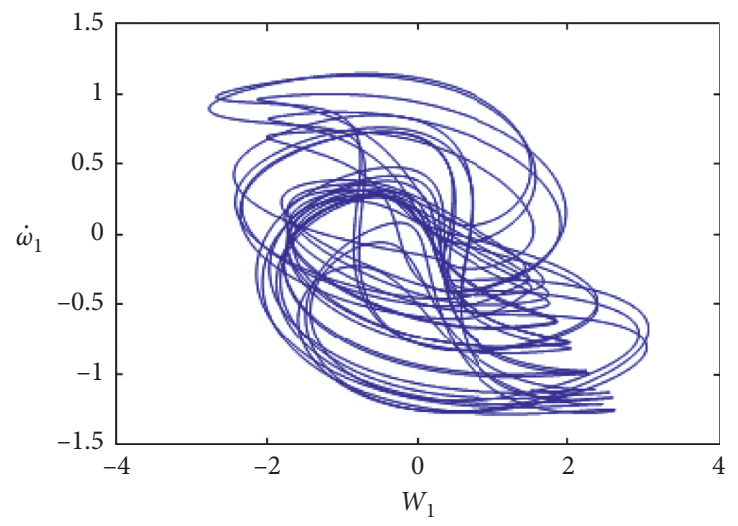

(c)

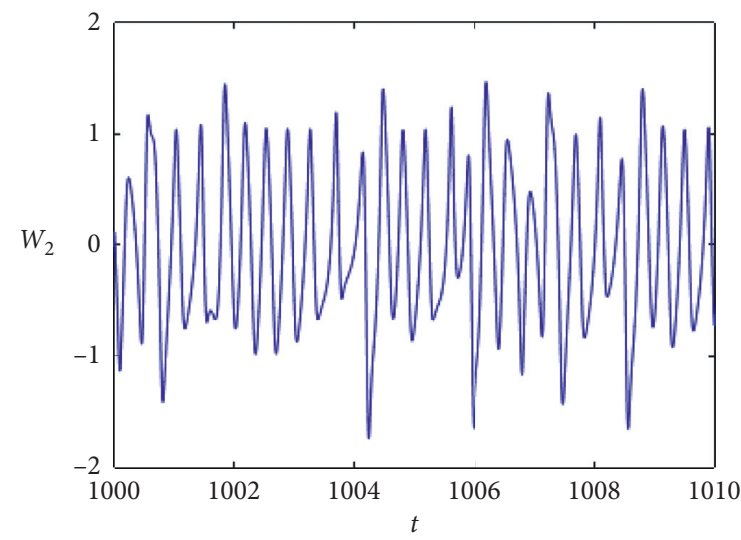

(b)

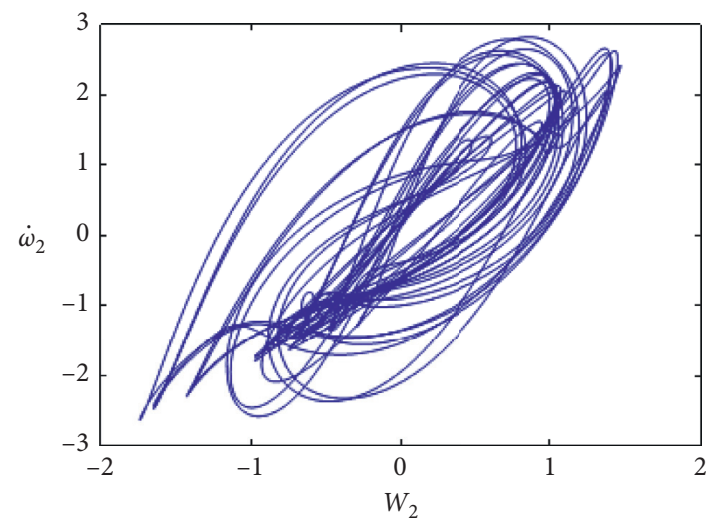

(d)

Figure 4: Continued. 


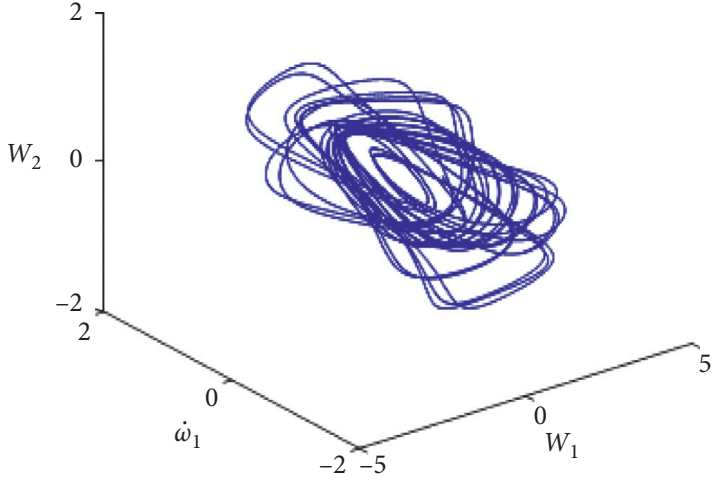

(e)

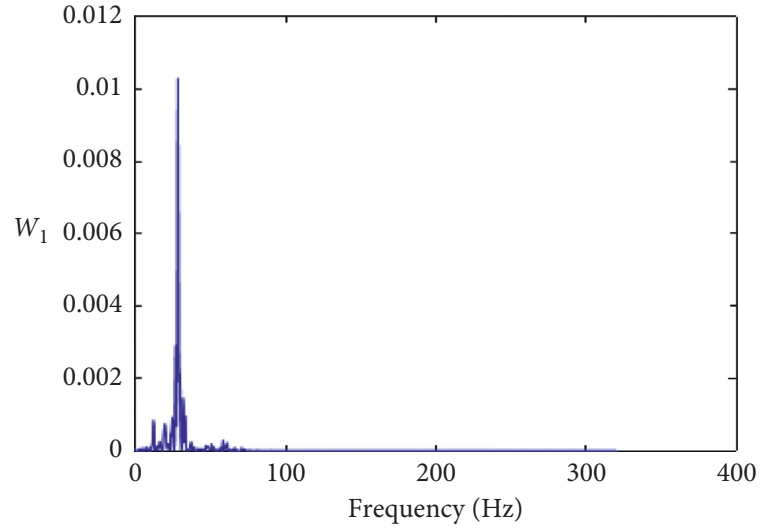

(f)

Figure 4: The motion of the blade with varying rotating speeds when $\Omega_{v}=1.8 \times 10^{2}(\mathrm{r} / \mathrm{min})$. The time history of the first-order mode of (a) $w_{1}$ and (b) $w_{2}$; the phase portrait (c) on plane $\left(w_{1}, \dot{w}_{1}\right)$, (d) on plane $\left(w_{2}, \dot{w}_{2}\right)$, and (e) in the three-dimensional space $\left(w_{1}, \dot{w}_{1}, w_{2}\right) ;(\mathrm{f})$ the PSD of $w_{1}$.

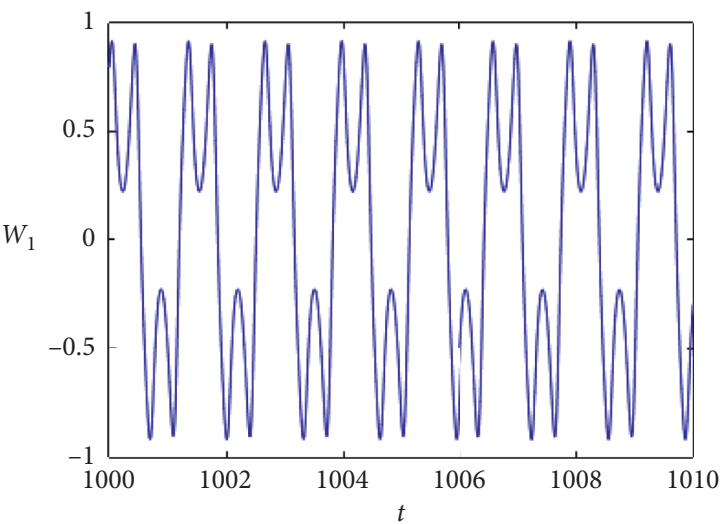

(a)

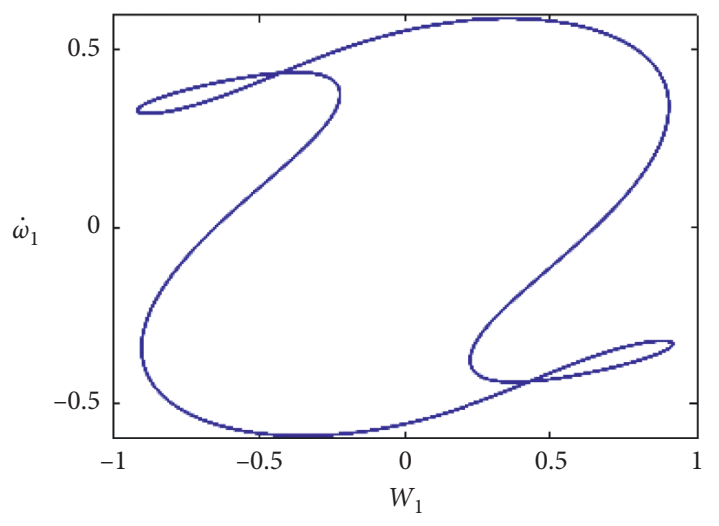

(c)

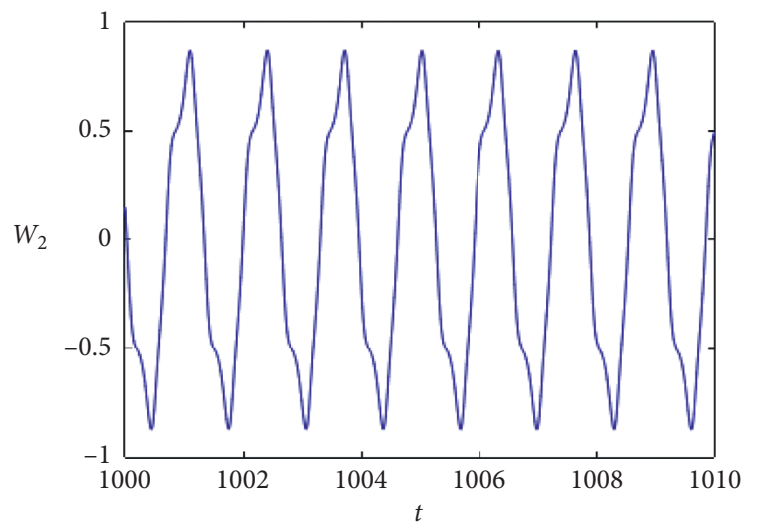

(b)

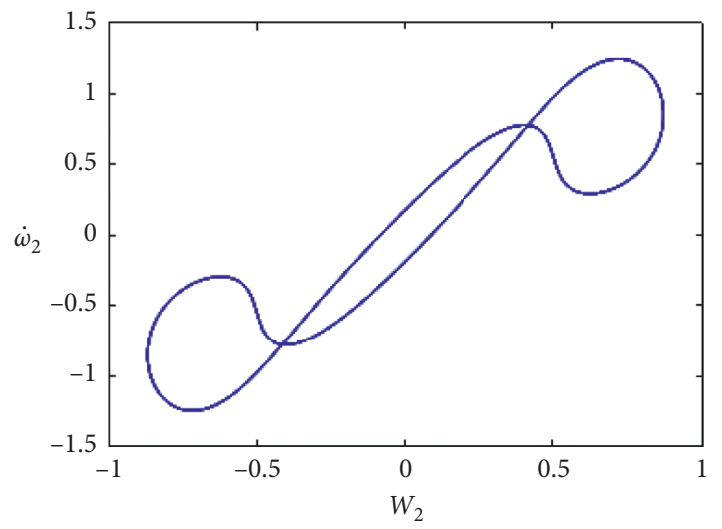

(d)

Figure 5: Continued. 


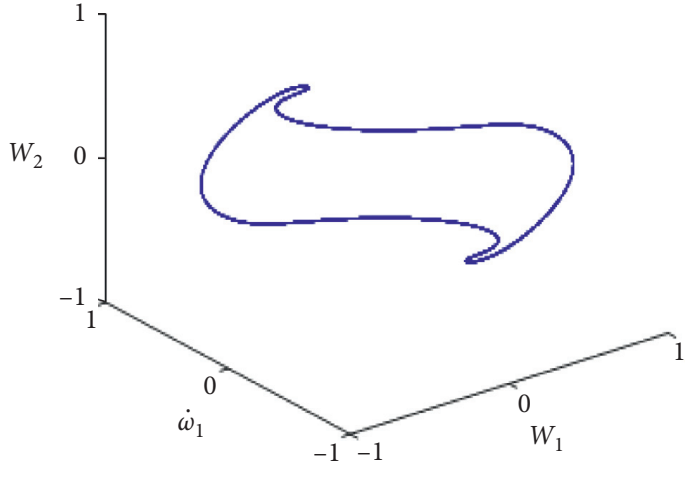

(e)

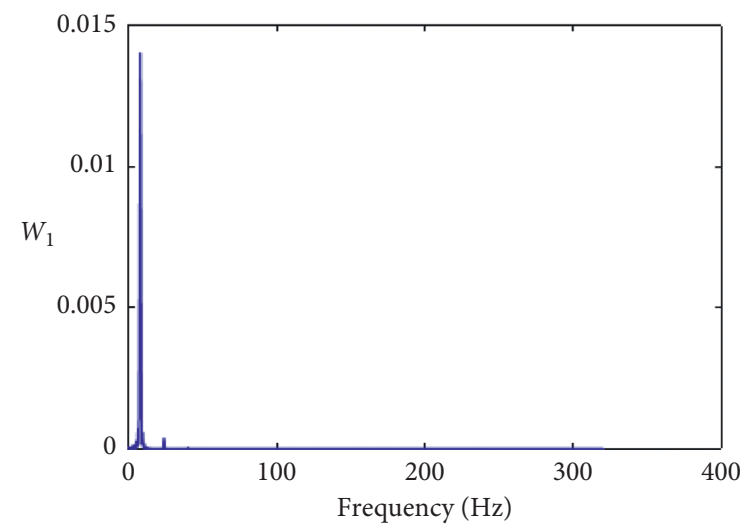

(f)

FIGURE 5: The motion of the blade with changing forces when $F_{1}=1.1 \times 10^{6}\left(\mathrm{~N} / \mathrm{m}^{2}\right)$. The time history of the first-order mode of (a) $w_{1}$ and (b) $w_{2}$; the phase portrait (c) on plane $\left(w_{1}, \dot{w}_{1}\right),(\mathrm{d})$ on plane $\left(w_{2}, \dot{w}_{2}\right)$, and (e) in the three-dimensional space $\left(w_{1}, \dot{w}_{1}, w_{2}\right)$; (f) the PSD of $w_{1}$.

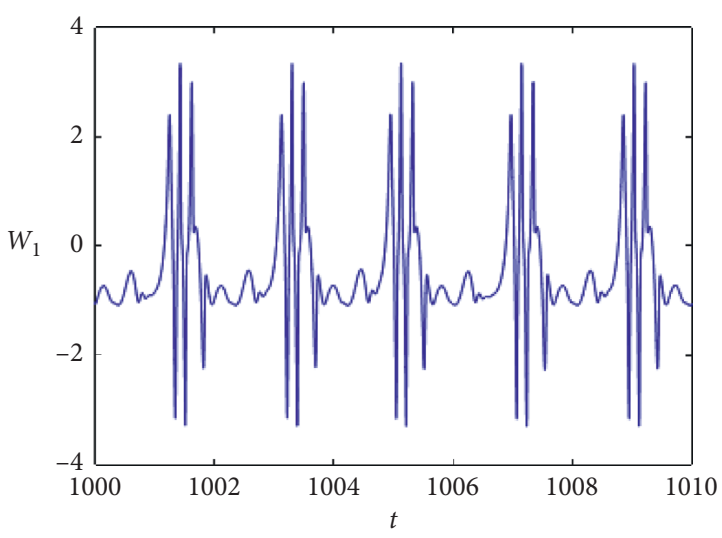

(a)

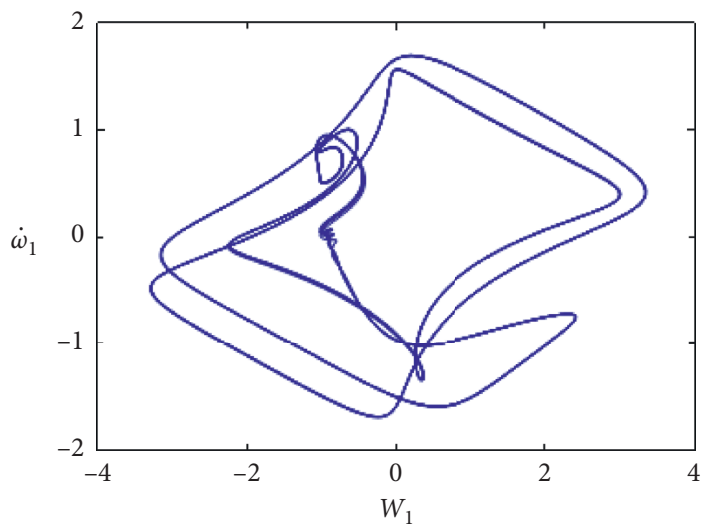

(c)

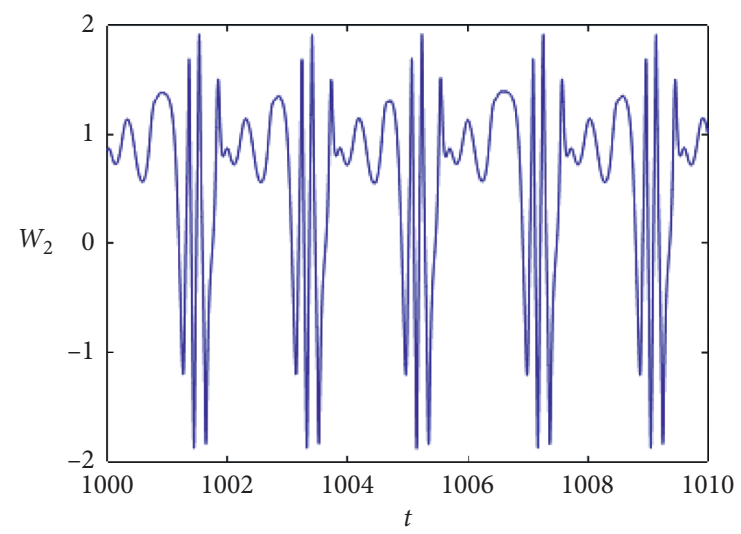

(b)

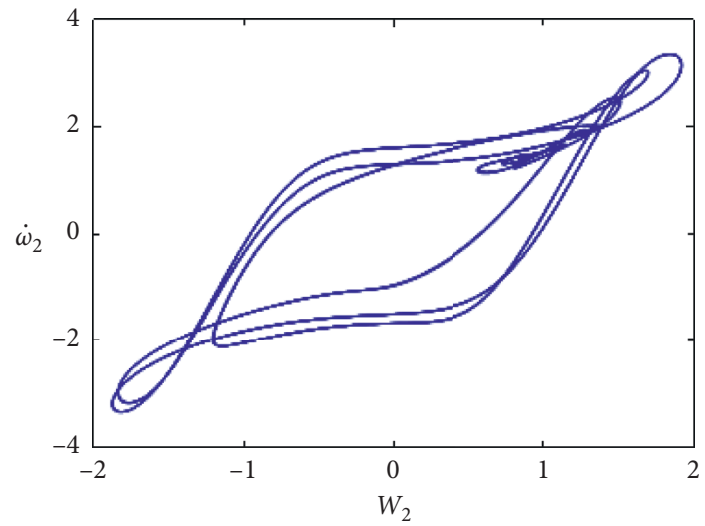

(d)

Figure 6: Continued. 


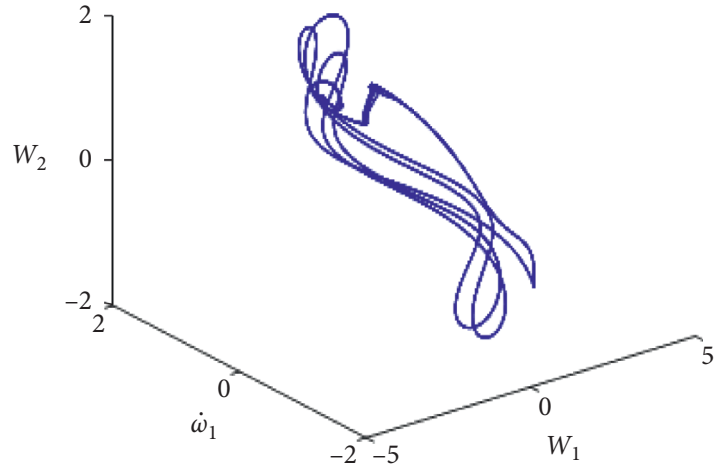

(e)

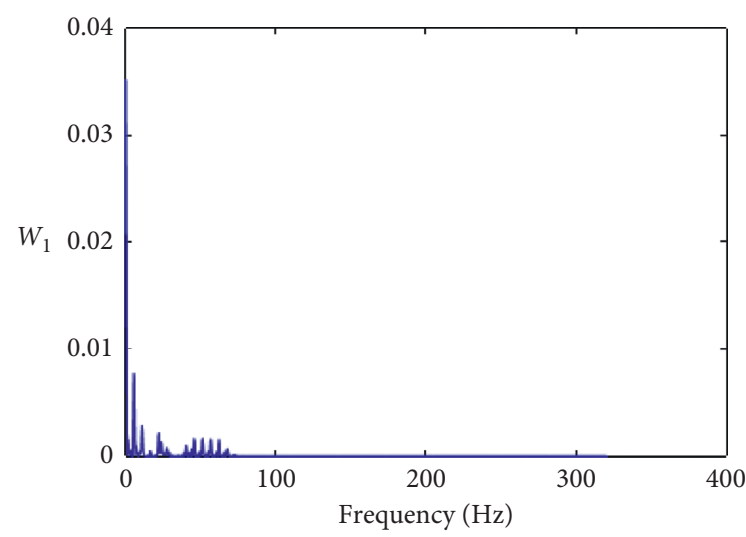

(f)

Figure 6: The motion of the blade with changing forces when $F_{1}=3.9 \times 10^{6}\left(\mathrm{~N} / \mathrm{m}^{2}\right)$. The time history of the first-order mode of (a) $w_{1}$ and (b) $w_{2}$; the phase portrait (c) on plane $\left(w_{1}, \dot{w}_{1}\right)$, (d) on plane $\left(w_{2}, \dot{w}_{2}\right)$, and (e) in the three-dimensional space $\left(w_{1}, \dot{w}_{1}, w_{2}\right)$; (f) the PSD of $w_{1}$.

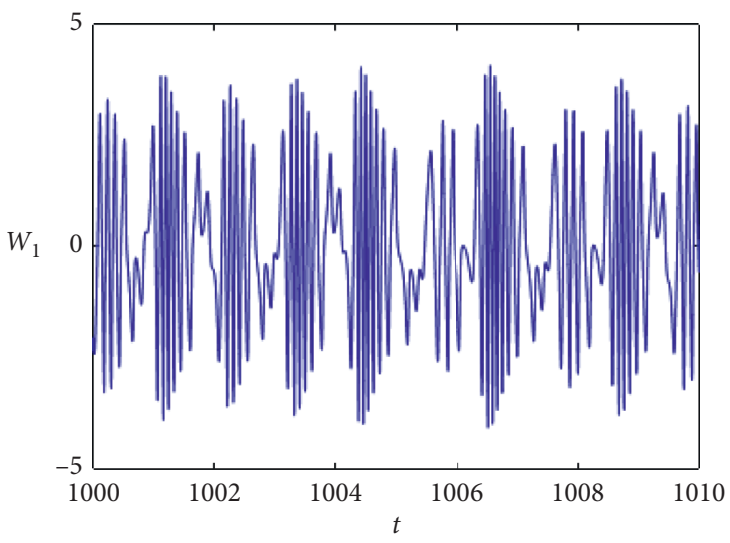

(a)

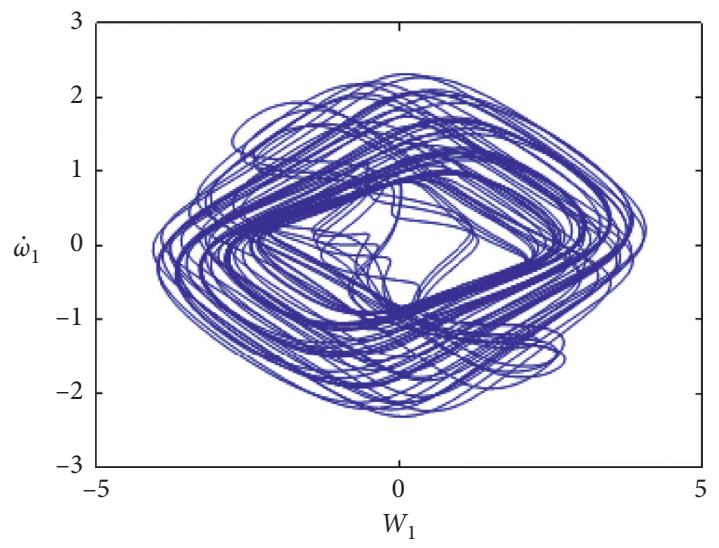

(c)

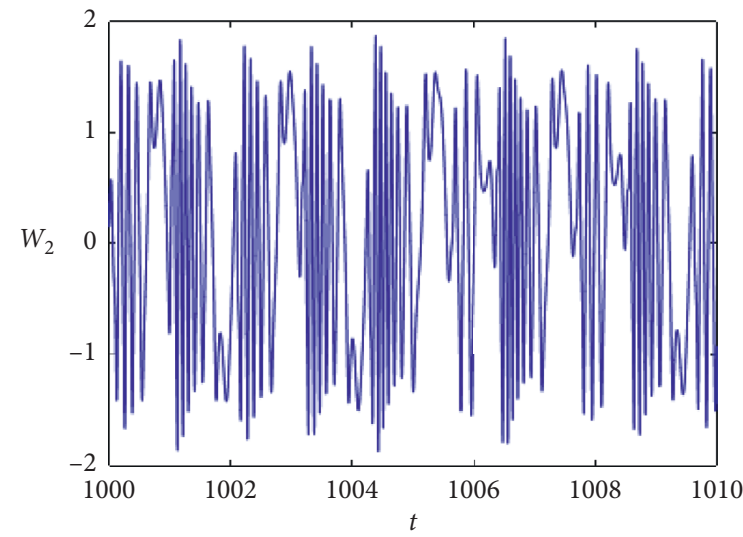

(b)

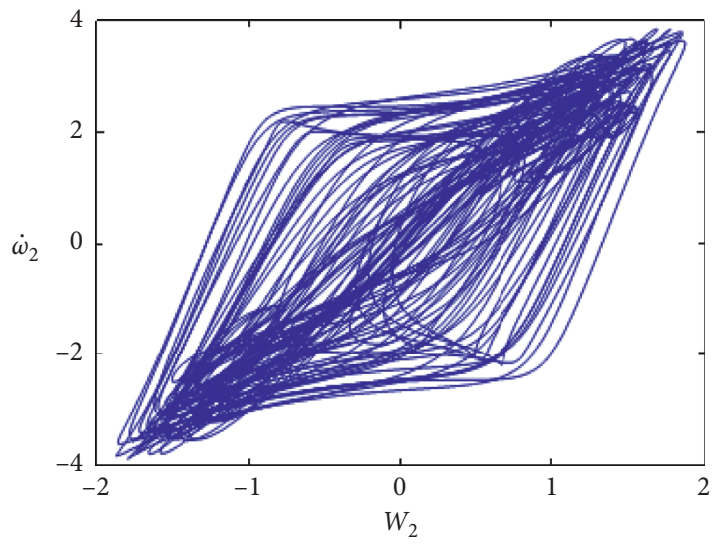

(d)

Figure 7: Continued. 


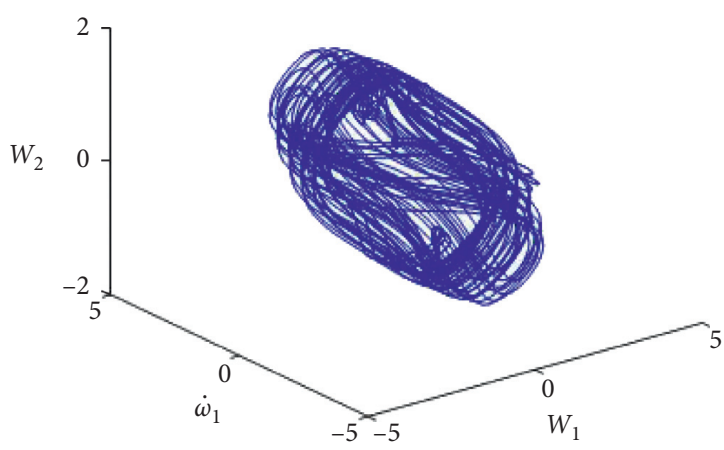

(e)

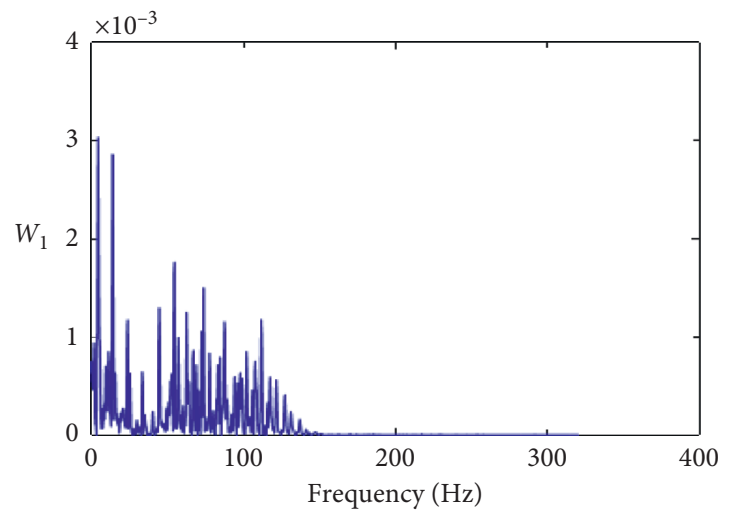

(f)

Figure 7: The motion of the blade with changing forces when $F_{1}=4.7 \times 10^{6}\left(\mathrm{~N} / \mathrm{m}^{2}\right)$. The time history of the first-order mode of (a) $w_{1}$ and (b) $w_{2}$; the phase portrait (c) on plane $\left(w_{1}, \dot{w}_{1}\right)$, (d) on plane $\left(w_{2}, \dot{w}_{2}\right)$, and (e) in the three-dimensional space $\left(w_{1}, \dot{w}_{1}, w_{2}\right)$; (f) the PSD of $w_{1}$.

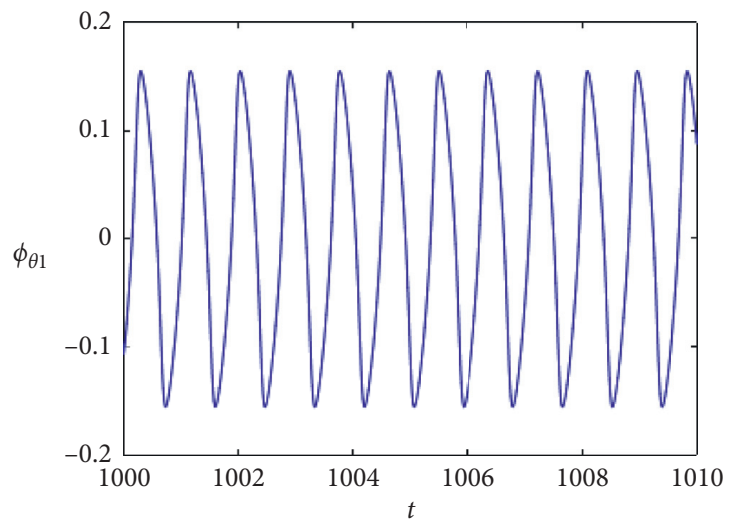

(a)

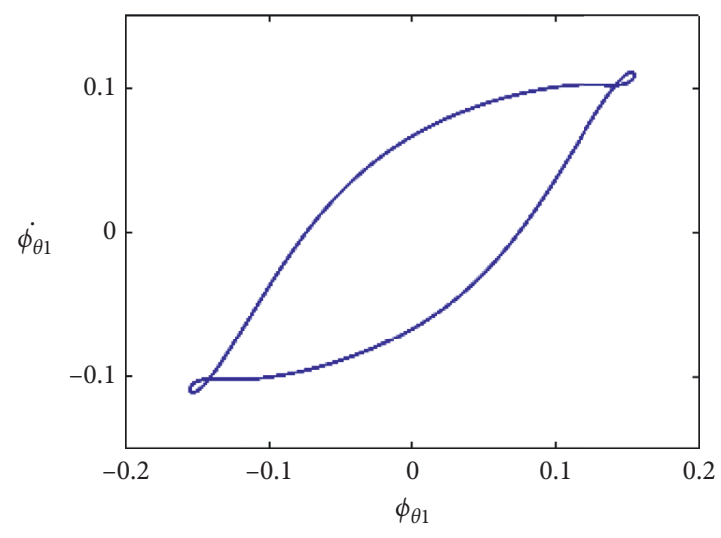

(c)

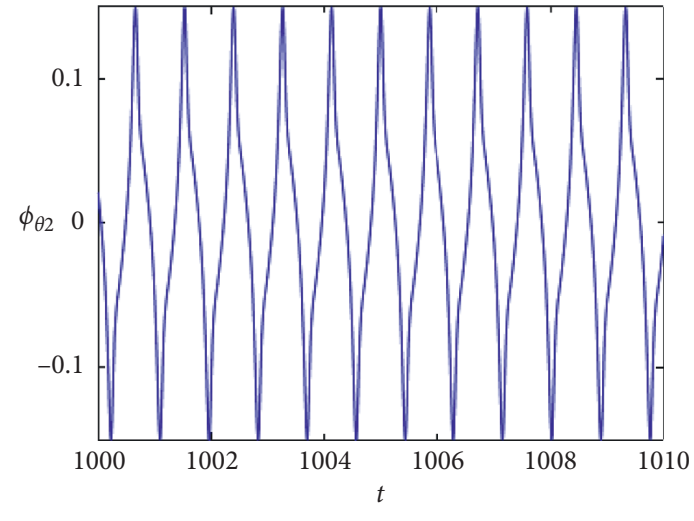

(b)

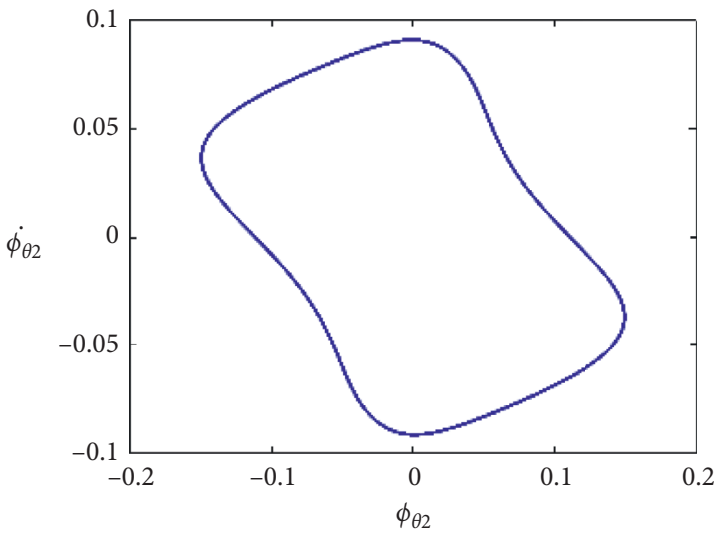

(d)

Figure 8: Continued. 


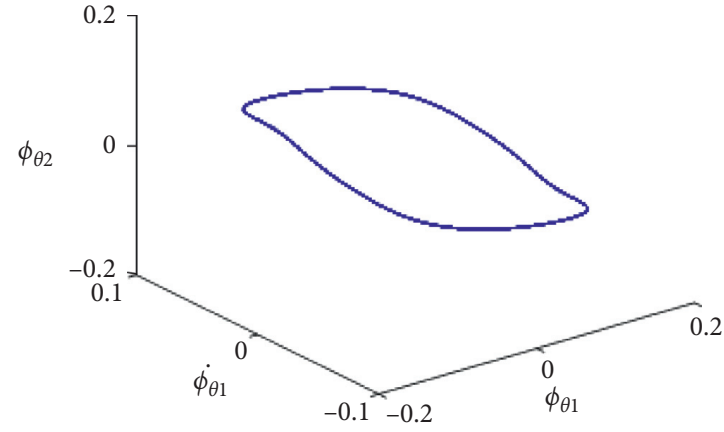

(e)

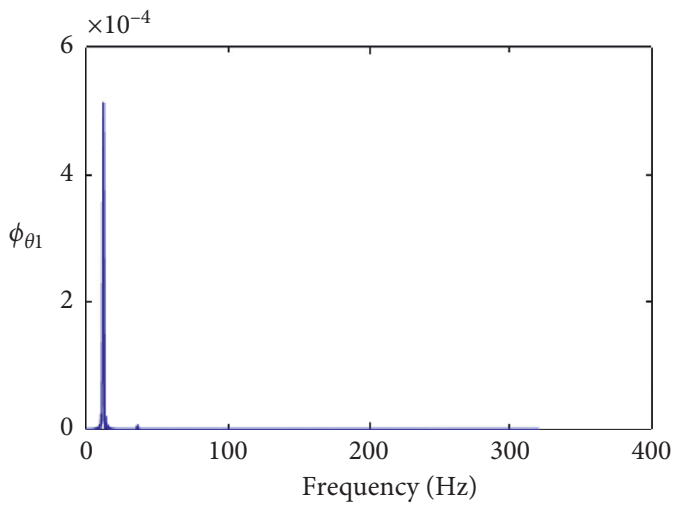

(f)

FIGURE 8: The motion of the blade with different moments when $M_{1}=0.89 \times 10^{6}(\mathrm{~N} / \mathrm{m})$. The time history of the first-order mode of (a) $\varphi_{\theta 1}$ and (b) $\varphi_{\theta 2}$; the phase portrait (c) on plane $\left(\varphi_{\theta 1}, \dot{\varphi}_{\theta 1}\right)$, (d) on plane $\left(\varphi_{\theta 2}, \dot{\varphi}_{\theta 2}\right)$, and (e) in the space $\left(\varphi_{\theta 1}, \dot{\varphi}_{\theta 1}, \varphi_{\theta 2}\right)$; (f) the PSD of $\varphi_{\theta 1}$.

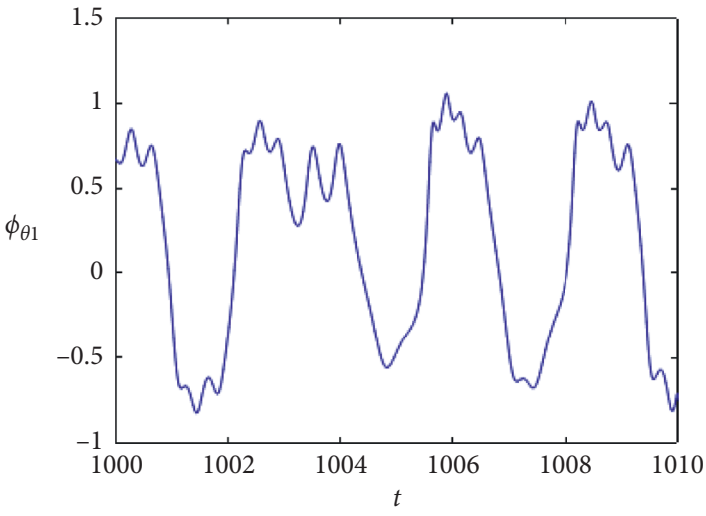

(a)

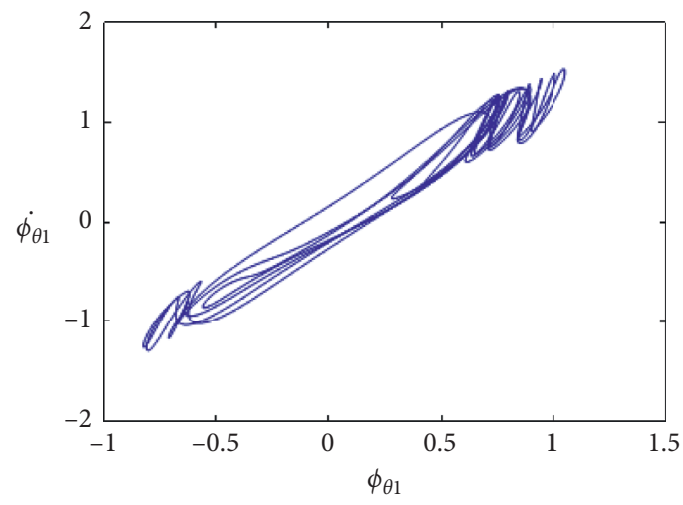

(c)

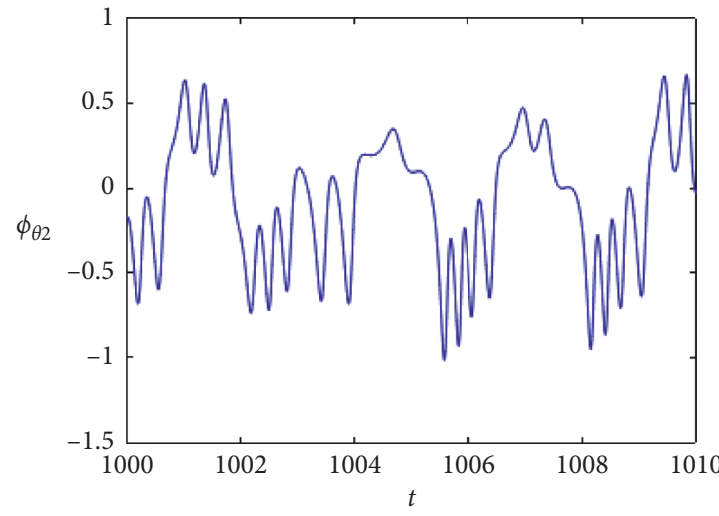

(b)

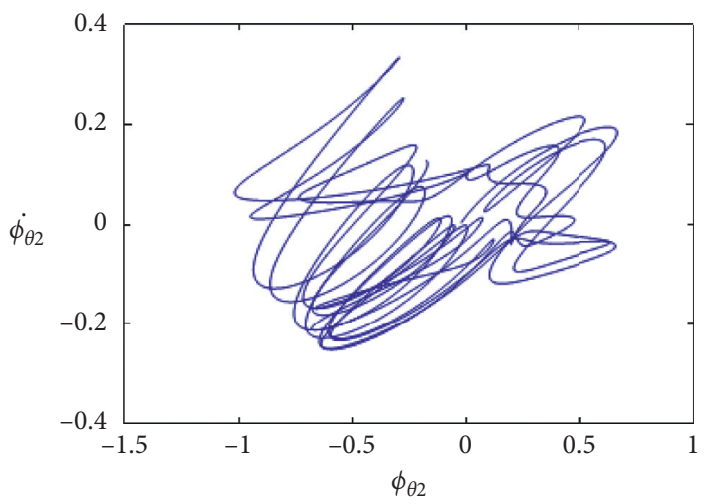

(d)

Figure 9: Continued. 


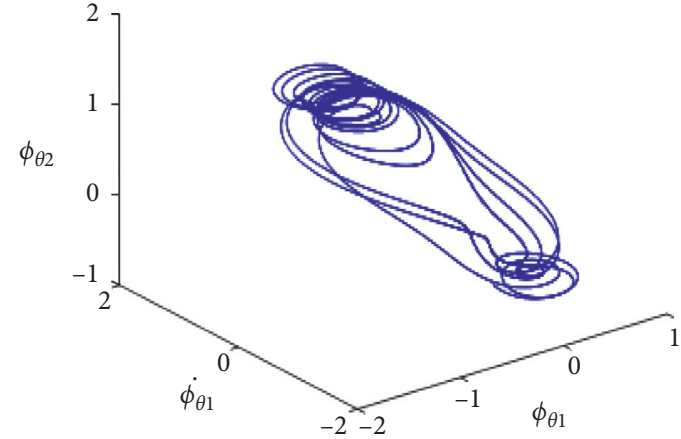

(e)

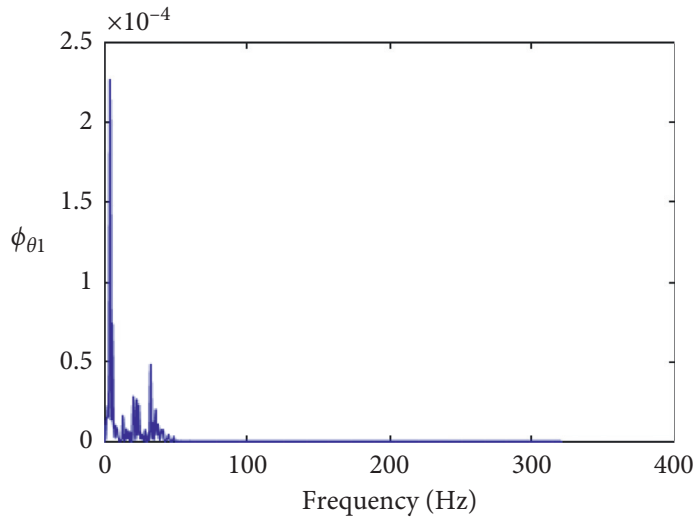

(f)

FIGURE 9: The motion of the blade with different moments when $M_{1}=3.2 \times 10^{6}(\mathrm{~N} / \mathrm{m})$. The time history of the first-order mode of (a) $\varphi_{\theta 1}$ and (b) $\varphi_{\theta 2}$; the phase portrait (c) on plane $\left(\varphi_{\theta 1}, \dot{\varphi}_{\theta 1}\right)$, (d) on plane $\left(\varphi_{\theta 2}, \dot{\varphi}_{\theta 2}\right)$, and (e) in the space $\left(\varphi_{\theta 1}, \dot{\varphi}_{\theta 1}, \varphi_{\theta 2}\right)$; (f) the PSD of $\varphi_{\theta 1}$.

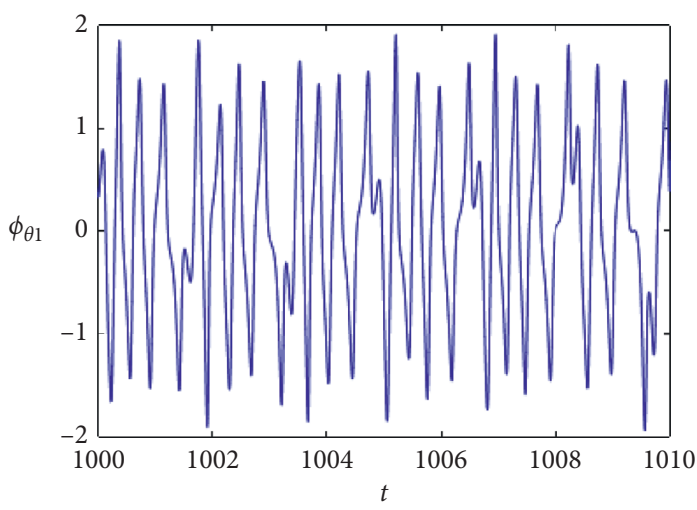

(a)

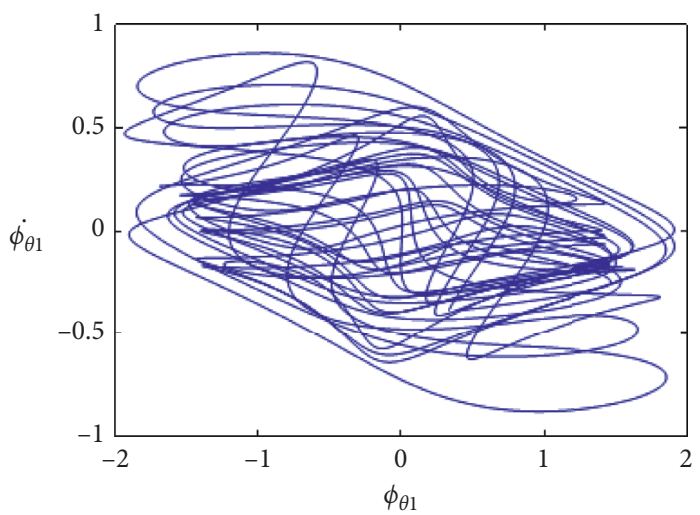

(c)

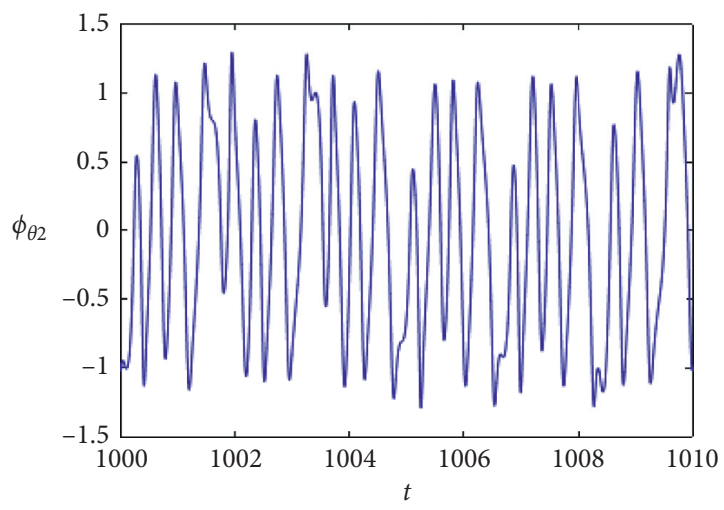

(b)

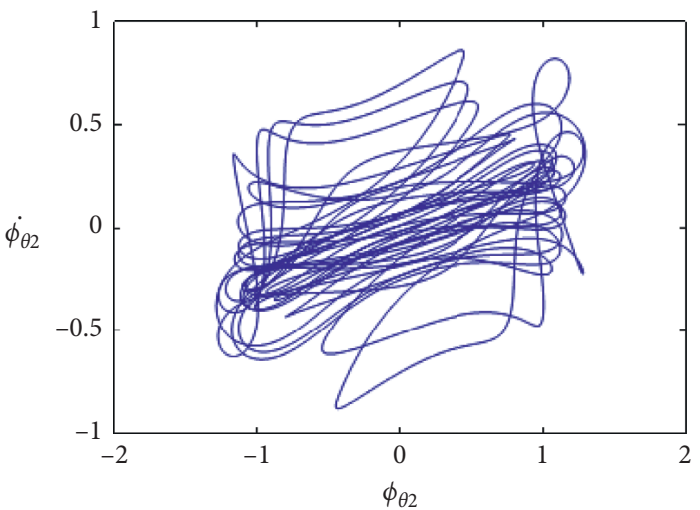

(d)

Figure 10: Continued. 


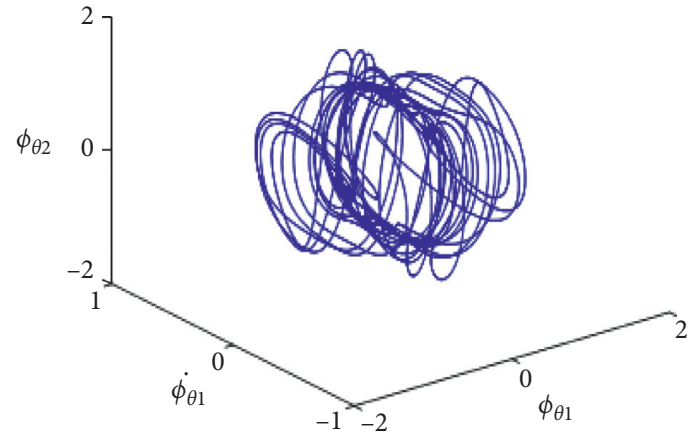

(e)

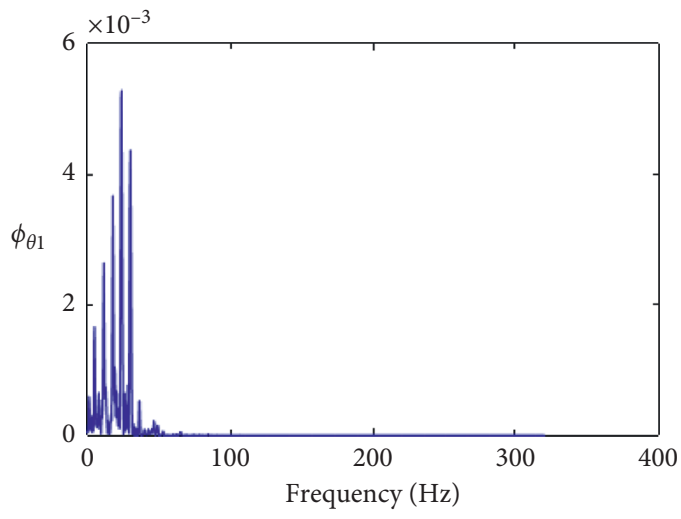

(f)

FIgURE 10: The motion of the blade with different moments when $M_{1}=4.0 \times 10^{6}(\mathrm{~N} / \mathrm{m})$. The time history of the first-order mode of (a) $\varphi_{\theta 1}$ and (b) $\varphi_{\theta 2}$; the phase portrait (c) on plane $\left(\varphi_{\theta 1}, \dot{\varphi}_{\theta 1}\right)$, (d) on plane $\left(\varphi_{\theta 2}, \dot{\varphi}_{\theta 2}\right)$, and (e) in the space $\left(\varphi_{\theta 1}, \dot{\varphi}_{\theta 1}, \varphi_{\theta 2}\right)$; (f) the PSD of $\varphi_{\theta 1}$.

$$
\begin{aligned}
L & =1.12 \mathrm{~m}, \\
r_{\text {root }} & =0.16 \mathrm{~m}, \\
\psi & =0.15 \pi, \\
\nu & =0.33, \\
h_{0} & =0.002 \mathrm{~m}, \\
g(\theta) & =1, \\
E & =70 \mathrm{GPa}, \\
\alpha & =\pi, \\
\rho & =2700\left(\mathrm{~kg} / \mathrm{m}^{3}\right) .
\end{aligned}
$$

3.1.2. Comparison with the Other Work. To validate the model proposed, we compare frequencies analytically calculated with frequencies obtained by the finite element method. Parameters of the blade are selected as

$$
\begin{aligned}
L & =0.15 \mathrm{~m}, \\
r_{\text {root }} & =0.05 \mathrm{~m}, \\
\psi & =\frac{\pi}{6}, \\
\nu & =0.33, \\
E & =102.04 \mathrm{GPa}, \\
h_{0} & =0.03 \mathrm{~m}, \\
g(\theta) & =1, \\
\alpha & =\frac{\pi}{9}, \\
\beta & =\frac{\pi}{6},
\end{aligned}
$$

$$
\begin{aligned}
\rho & =4450\left(\mathrm{~kg} / \mathrm{m}^{3}\right) \\
\kappa & =300(\mathrm{Ns} / \mathrm{m}), \\
\gamma & =300(\mathrm{Ns} / \mathrm{m}) \\
F_{0} & =1.3 \times 10^{6}\left(\mathrm{~N} / \mathrm{m}^{2}\right), \\
M_{0} & =1.1 \times 10^{6}(\mathrm{~N} / \mathrm{m}), \\
K_{u_{1}} & =0.59, K_{w}
\end{aligned}
$$

Tables 1 and 2 show the frequencies of different orders obtained by the methods above. The model is reasonable because the frequencies calculated are in good agreement.

3.2. Effect of Varying Velocities. In what follows, the parameters and the initial conditions are chosen as

$$
\begin{aligned}
L & =0.21 \mathrm{~m}, \\
r_{\text {root }} & =0.04 \mathrm{~m}, \\
\psi & =\frac{\pi}{6}, \\
\nu & =0.33, \\
E & =102.04 \mathrm{GPa}, \\
h_{0} & =0.05 \mathrm{~m}, \\
\omega_{r} & =100, \\
\gamma & =300(\mathrm{Ns} / \mathrm{m}), \\
\kappa & =300(\mathrm{Ns} / \mathrm{m}), \\
K_{u_{1}} & =0.59 \\
\alpha & =\frac{\pi}{9},
\end{aligned}
$$




$$
\begin{aligned}
\beta & =\frac{\pi}{6}, \\
\rho & =4450\left(\mathrm{~kg} / \mathrm{m}^{3}\right), \\
K_{w} & =0.62, \\
F_{0} & =1.5 \times 10^{6}\left(\mathrm{~N} / \mathrm{m}^{2}\right), \\
M_{0} & =1.2 \times 10^{6}(\mathrm{~N} / \mathrm{m}), \\
\Omega_{c} & =2000(\mathrm{r} / \mathrm{min}) .
\end{aligned}
$$

The chaotic and periodic responses can be identified by several conventional criteria. The waveform, phase portraits, and the power spectrum are utilized to verify the existence of the chaotic and periodic motion of the blade.

Under the different rotating speeds $\Omega_{v}=0.17,1.09$, and $1.8 \times 10^{2}(\mathrm{r} / \mathrm{min})$, Figures $2-4$ show the period- 1 motion, multiperiod motion, and chaotic motion of the cantilever conical shell, respectively. It is observed that the rotating speed $\Omega_{v}$ makes great difference on the nonlinear dynamic responses of the cantilever shell.

Comparing Figures 2-4, one finds that both of the maximum amplitudes of $w_{1}$ and $w_{2}$ are increasing with the increasing rotating speed. Namely, the vibration mode changes as the increase of the rotating speed.

3.3. Effect of Changing Forces. The parameters of the conical shell and the initial conditions are chosen as

$$
\begin{aligned}
L & =0.19 \mathrm{~m}, \\
r_{\text {root }}= & 0.03 \mathrm{~m}, \\
\alpha & =\frac{\pi}{9} \\
\beta & =\frac{\pi}{6} \\
\omega_{r} & =97, \\
& \rho=4450\left(\mathrm{~kg} / \mathrm{m}^{3}\right), \\
\kappa & =300\left(\mathrm{Ns} / \mathrm{m}^{3},\right. \\
\psi= & \frac{\pi}{6} \\
\nu= & 0.33, \\
h_{0}= & 0.033 \mathrm{~m}, \\
K_{u_{1}} & =0.49, \\
E & =102.04 \mathrm{GPa}, \\
\gamma= & 300(\mathrm{Ns} / \mathrm{m}), \\
K_{w}= & 0.57, \\
F_{0}= & 1.66 \times 10^{6}\left(\mathrm{~N} / \mathrm{m}^{2}\right), \\
\Omega_{c}= & 1600(\mathrm{r} / \mathrm{min}), \\
M_{0}= & 1.04 \times 10^{6}\left(\mathrm{~N} / \mathrm{m}^{2},\right.
\end{aligned}
$$

Nonlinear oscillation of the system is investigated by choosing the rotating speed $f_{1}$ as the controlling parameter. Figures 5-7 present the period-1 motion, multiperiod motion, and chaotic motion of the rotating conical shell with the different force $f_{1}=1.1,3.9$ and $4.7 \times 10^{6}\left(\mathrm{~N} / \mathrm{m}^{2}\right)$, respectively. It can be shown from figures that the amplitude of the first-order mode is larger than that of the second-order mode. The amplitudes of $w_{1}$ and $w_{2}$, the distribution of the PSD, and phase portraits are very sensitive to the value of the force $f_{1}$.

3.4. Effect of Different Moments. The parameters and the initial conditions are chosen as

$$
\begin{aligned}
& L=0.27 \mathrm{~m} \text {, } \\
& r_{\text {root }}=0.04 \mathrm{~m} \text {, } \\
& \psi=\frac{\pi}{6} \\
& \alpha=\frac{\pi}{9}, \\
& E=102.04 \mathrm{GPa} \text {, } \\
& v=0.33 \\
& h_{0}=0.03 \mathrm{~m} \\
& \rho=4450\left(\mathrm{~kg} / \mathrm{m}^{3}\right), \\
& \kappa=300(\mathrm{Ns} / \mathrm{m}), \\
& \omega_{r}=90 \text {, } \\
& \gamma=300(\mathrm{Ns} / \mathrm{m}) \\
& F_{0}=1.4 \times 10^{6}\left(\mathrm{~N} / \mathrm{m}^{2}\right) \text {, } \\
& M_{0}=1.1 \times 10^{6}(\mathrm{~N} / \mathrm{m}) \text {, } \\
& \Omega_{c}=1570(\mathrm{r} / \mathrm{min}) .
\end{aligned}
$$

$K_{u_{1}}=0.58$ and $K_{w}=0.6$. When other parameters and the initial condition do not vary, we only change $m_{1}$ to detect the influence of the moment on vibration of the rotating cantilever blade. As the rotating speed and the force shown in the figures above, the moment $m_{1}$ also plays an important role for the nonlinear dynamic responses of the cantilever shell. Figures 8-10 contrast the period-1 motion, multiperiod motion, and chaotic motion in the different value of external moment $m_{1}=0.89,3.2$ and $4.0 \times 10^{6}(\mathrm{~N} / \mathrm{m})$, respectively. These figures indicate that the amplitude, the phase portraits, and the power spectrum densities vary from the different value of the external moment. The amplitude of the rotating cantilever conical shell increases with the increase of the moment. 


\section{Conclusions}

In order to describe the nonlinear dynamic responses of the blade with variable thickness, this paper presents a rotating pretwisted cantilever conical shell with variable thickness to investigate the effects of the varying rotating speed, external force, and external moment on the dynamic behaviors. Based on the von Kármán nonlinear relationship, Hamilton's principle, and the first-order shear deformation theory, the nonlinear governing equations of motion for the rotating cantilever blade with variable thickness are established. Galerkin's method is utilized to discretize the partial differential governing equations of motion as a nonlinear ordinary differential equation of motion. Numerical results show that the rotating velocity, external force, and moment have significant influences on nonlinear dynamic behaviors of the cantilever conical shell. It can be concluded as follows:

(1) The numerical results demonstrate that nonlinear dynamic motions of the blade are sensitive to the rotating speed. The increase of the rotating speed could not only change the distribution of the PSD and the phase portraits but also initiate the amplitude increase. With the increasing rotating speed, the periodic motion, and chaotic motion of the blade which appears, the motion law of the rotating blade performs as follows: period-1 motion $\longrightarrow$ multiperiod motion $\longrightarrow$ chaotic motion. Occurrence of the chaotic motion means that the rotating cantilever blade may perform large amplitude nonlinear vibration, which leads to the damage of the system. We can control the responses of the system from the chaotic motions to the periodic motions by changing the rotating speed.

(2) The phase portrait and power spectrum are conducted to demonstrate that periodic motions and chaotic motions occur in nonlinear vibrations of the rotating blade under certain conditions. The increase of forces and moments could not only change the distribution of the PSD and the phase portraits but also initiate the amplitude increase. With the increasing external excitation, the motion law of the rotating blade performs as follows: period-1 motion $\longrightarrow$ multiperiod motion $\longrightarrow$ chaotic motion. Since chaotic motion is the large amplitude nonlinear vibration, we can adjust the external force and external moment to control the responses of the system in order to avoid the appearance of the chaotic motion.

Study on nonlinear behavior of the blade is of great significance. Our results are useful to improve the stability of the rotating blade.

\section{Data Availability}

The data used to support the findings of this study are available from the corresponding author upon request.

\section{Conflicts of Interest}

The authors declare that there are no conflicts of interest regarding the publication of this article.

\section{Acknowledgments}

The authors acknowledge the financial support of the $\mathrm{Na}$ tional Natural Science Foundation of China through grant nos. 11290152 and 11427801.

\section{References}

[1] H. Qinkai and C. Fulei, "Parametric instability of a rotating truncated conical shell subjected to periodic axial loads," Mechanics Research Communication, vol. 53, pp. 63-74, 2013.

[2] P. Malekzadeh and Y. Heydarpour, "Free vibration analysis of rotating functionally graded truncated conical shells," Composite Structures, vol. 97, pp. 76-188, 2013.

[3] J. E. Goldberg, J. L. Bogdanoff, and L. Marcus, "On the calculation of the axisymmetric modes and frequencies of conical shells," The Journal of the Acoustical Society of America, vol. 32, no. 6, pp. 738-742, 1960.

[4] C. C. Yang, "On vibrations of orthotropic conical shells," Journal of Sound and Vibration, vol. 34, no. 4, pp. 552-555, 1974.

[5] R. S. Srinivasan and P. A. Krishnan, "Dynamic response of layered conical shell panel using integral equation technique," Computers \& Structures, vol. 31, no. 6, pp. 897-905, 1989.

[6] K. M. Liew, C. W. Lim, and L. S. Ong, "Vibration of pretwisted cantilever shallow conical shells," International Journal of Solids and Structures, vol. 31, no. 18, pp. 2463-2476, 1994.

[7] O. G. Mcgee and H. R. Chu, "Three-dimensional vibration analysis of rotating laminated composite blades," Journal of Engineering for Gas Turbines and Power, vol. 116, no. 3, pp. 663-671, 1994.

[8] J. J. Lee, C. H. Yeom, and I. Lee, "Vibration analysis of twisted cantilevered conical composite shells," Journal of Sound and Vibration, vol. 255, no. 5, pp. 965-982, 2002.

[9] N. V. S. Naidu and P. K. Sinha, "Nonlinear finite element analysis of laminated composite shells in hygrothermal environments," Composite Structures, vol. 69, no. 4, pp. 387-395, 2005.

[10] S. Kumari and P. K. Sinha, "Hygrothermal analysis of composite wing T-joints," Aircraft Engineering and Aerospace Technology, vol. 74, no. 1, pp. 23-37, 2002.

[11] A. Ghosh, "Hygrothermal effects on the initiation and propagation damage in composite shells," Aircraft Engineering and Aerospace Technology, vol. 80, no. 4, pp. 386-399, 2008.

[12] T. Bandyopadhyay, A. Karmakar, and K. Kishimoto, "Hygrothermal effects on the free vibration characteristics of delaminated composite pretwisted rotating conical shells," Mechanical Engineering Journal, vol. 1, no. 6, pp. 1-18, 2014.

[13] C. Hwu, H. W. Hsu, and Y. H. Lin, "Free vibration of composite sandwich plates and cylindrical shells," Composite Structures, vol. 171, pp. 528-537, 2017.

[14] A. K. Garg, R. K. Khare, and T. Kant, "Higher-order closedform solutions for free vibration of laminated composite and sandwich shells," Journal of Sandwich Structures \& Materials, vol. 8, no. 3, pp. 205-235, 2006.

[15] O. Rahmani, S. M. R. Khalili, and K. Malekzadeh, "Free vibration response of composite sandwich cylindrical shell with 
flexible coreflexible core," Composite Structures, vol. 92, no. 5, pp. 1269-1281, 2010.

[16] A. Kumar, A. Chakrabarti, and P. Bhargava, "Vibration of laminated composites and sandwich shells based on higher order zigzag theory," Engineering Structures, vol. 56, pp. 880-888, 2013.

[17] C. W. Lim, K. M. Liew, and S. Kitipornchai, "Vibration of cantilevered laminated composite shallow conical shells," International Journal of Solids and Structures, vol. 35, no. 15, pp. 1695-1707, 1998.

[18] A. H. Sofyev, "Application of the first order shear deformation theory to the solution of free vibration problem for laminated conical shells," Composite Structure, vol. 188, pp. 340-346, 2018.

[19] A. H. Sofyev, "Review of research on the vibration and buckling of the FGM conical shells," Composite Structures, vol. 211, pp. 301-317, 2019.

[20] D. J. Wilkins Jr., C. W. Bert, and D. M. Egle, "Free vibrations of orthotropic sandwich conical shells with various boundary conditions," Journal of Sound and Vibration, vol. 13, no. 2, pp. 211-228, 1970.

[21] N. S. Bardell, J. M. Dunsdon, and R. S. Langley, "Free vibration of thin, isotropic, open, conical panels," Journal of Sound and Vibration, vol. 217, no. 2, pp. 297-320, 1998.

[22] M. Nasihatgozar and S. Khalili, "Vibration and buckling analysis of laminated sandwich conical shells using higher order shear deformation theory and differential quadrature methodfferential quadrature method," Journal of Sandwich Structures \& Materials, vol. 21, no. 4, pp. 1445-1480, 2019.

[23] A. H. Sofyev and E. Osmancelebioglu, "The free vibration of sandwich truncated conical shells containing functionally graded layers within the shear deformation theory," Composites Part B, vol. 120, pp. 197-211, 2017.

[24] T. B. Singhaa, M. Routb, T. Bandyopadhyayc, and K. Amit, "Free vibration analysis of rotating pretwisted composite sandwich conical shells with multiple debonding in hygrothermal environment," Engineering Structures, vol. 204, pp. 110058-110061, 2020.

[25] M. Jakomin and F. Kosel, "Stability of the shallow axisymmetric parabolic-conic bimetallic shell by nonlinear theory," Mathematical Problems in Engineering, vol. 2011, Article ID 145638, 30 pages, 2011.

[26] F. M. A. da Silva, A. F. Brazão, and P. B. Gonçalves, "Influence of physical and geometrical uncertainties in the parametric instability load of an axially excited cylindrical shell," Mathematical Problems in Engineering, vol. 2015, Article ID 758959, 18 pages, 2015.

[27] X.-Q. Li, W. Zhang, X.-D. Yang, and L.-K. Song, "A unified approach of free vibration analysis for stiffened cylindrical shell with general boundary conditions," Mathematical Problems in Engineering, vol. 2019, Article ID 4157930, 14 pages, 2019.

[28] M. M. H. Mirzaei, M. Arefi, and A. Loghman, "Thermoelastic analysis of a functionally graded simple blade using first-order shear deformation theory," Mechanics of Advanced Composite Structures, vol. 2014, pp. 1-10, 2014.

[29] M. M. H. Mirzaei, M. Arefi, and A. Loghman, "Time-dependent creep analysis of a functionally graded simple blade using first-order shear deformation theory," Autralian Journal of Mechanical Engineering, vol. 2019, pp. 1-13, 2019.

[30] M. H. Yao, Y. P. Chen, and W. Zhang, "Nonlinear vibrations of blade with varying rotating speed," Nonlinear Dynamics, vol. 68, no. 4, pp. 487-504, 2012.
[31] F. Wang and W. Zhang, "Stability analysis of a nonlinear rotating blade with torsional vibrations," Journal of Sound and Vibration, vol. 331, no. 26, pp. 5755-5773, 2012.

[32] N. F. Rieger, Rotordynamics 2 Problems in Turbomachinery, Springer-Verlag Wien, New York, NY, USA, 1988.

[33] S. W. Yang, Y. X. Hao, W. Zhang, and S. B. Li, "Nonlinear dynamic behavior of functionally graded truncated conical shell under complex loads," International Journal of Bifurcation and Chaos, vol. 25, no. 2, pp. 11053-11061, 2015.

[34] A. H. Nayfeh and D. T. Mook, Nonlinear Oscillations, WileyInterscience, New York, NY, USA, 1979.

[35] M. Tong, S. G. Li, C. J. Lu, and Z. J. Luo, "Vibration characteristic analysis of aero-engine first stage fan blades," Aviation Maintenance \& Engineering, vol. 2016, pp. 63-66, 2016.

[36] T. Ye, G. Jin, Z. Su, and X. Jia, "A unified Chebyshev-Ritz formulation for vibration analysis of composite laminated deep open shells with arbitrary boundary conditions," Archive of Applied Mechanics, vol. 84, no. 4, pp. 441-471, 2014. 